\title{
JNK1 Inhibits GluR1 Expression and GluR1-Mediated Calcium Influx through Phosphorylation and Stabilization of Hes-1
}

\author{
Cheng H. Lin ${ }^{1}$ and Eminy H. Y. Lee ${ }^{2}$ \\ ${ }^{1}$ Graduate Institute of Life Sciences, National Defense Medical Center and ${ }^{2}$ Institute of Biomedical Sciences, Academia Sinica, Taipei 115, Taiwan
}

The GluR1 subunit of the AMPA receptor plays an important role in excitatory synaptic transmission and synaptic plasticity in the brain, but the regulation mechanism for GluR1 expression is largely unknown. Hairy and enhancer of split 1 (Hes-1) is a mammalian transcription repressor that regulates neuronal differentiation and development, but the role of Hes-1 in differentiated neurons is also less known. Here, we examined the molecular mechanism in regulation of GluR1 expression in rat cultured cortical neurons. We found that Hes-1 suppressed GluR1 promoter activity and decreased GluR1 expression through direct binding to the N-box and through preventing Mash1/E47 from binding to the E-box of GluR1 promoter. We also found that Hes-1 could be regulated by c-Jun N-terminal kinase (JNK1). JNK1 directly phosphorylates Hes-1 at Ser-263. Furthermore, JNK1 phosphorylation of Hes-1 stabilized the Hes-1 protein and enhanced the suppressing effect of Hes-1 on GluR1 expression. These effects were demonstrated both in the soma and at the synapse. Moreover, this JNK1-mediated signaling pathway was found to inhibit AMPA-evoked calcium influx in cortical neurons and this regulation mechanism is Notch independent. Here, we provided the first evidence that Hes-1 plays an important role in synaptic function in differentiated neurons. We also identified a novel JNK1-Hes-1 signaling pathway that regulates GluR1 expression involved in synaptic function in rat cortical neurons.

\section{Introduction}

Glutamate is a major excitatory neurotransmitter in the brain, and the AMPA receptor is a subtype of glutamate receptor that mediates most fast excitatory synaptic transmission in the brain (Dingledine et al., 1999). It also plays an important role in synaptic plasticity (Malinow and Malenka, 2002). The AMPA receptor is a homotetramer or heterotetramer protein consisting of subunit GluR1-4 (Hollmann and Heinemann, 1994). Upon presynaptic glutamate release, GluR1 is the key subunit that drives AMPA receptors to the synapse to activate NMDA receptor (Shi et al., 2001). It is found that the GluR1-containing AMPA receptors are rapidly incorporated into synapses during hippocampal long-term potentiation (LTP) (Makino and Malinow, 2009). Other evidence further suggests that GluR1 mediates the effect of AMPA receptor activation on synaptic plasticity because mice lacking GluR1 show impaired hippocampal LTP (Zamanillo et al., 1999; Mack et al., 2001) and reexpression of GluR1 in CA1 neurons restores this impairment (Mack et al., 2001). Despite the

Received July 4, 2011; revised Dec. 5, 2011; accepted Dec. 9, 2011.

Author contributions: C.H.L. and E.H.Y.L. designed research; C.H.L. performed research; C.H.L. analyzed data; C.H.L. and E.H.Y.L. wrote the paper.

This work was supported by National Science Council of Taiwan Grants NSC100-2321-B-001-019 and NSC99-2321-B-001-023. We thank Drs. Yi-Ping Hsueh and Yi-Shuian Huang for providing some cultured neurons.

The authors declare no competing financial interests.

Correspondence should be addressed to Dr. Eminy H. Y. Lee, Institute of Biomedical Sciences, Academia Sinica, Taipei 115, Taiwan. E-mail: eminy@gate.sinica.edu.tw.

DOI:10.1523/JNEUROSCI.3380-11.2012

Copyright $\odot 2012$ the authors $\quad 0270-6474 / 12 / 321826-21 \$ 15.00 / 0$ fact that GluR1 plays an important role in synaptic plasticity, the molecular mechanism in regulation of GluR 1 expression is barely known. The present study was aimed to address this issue.

Hairy and enhancer of split 1 (Hes-1) is a mammalian basic helix-loop-helix (bHLH) factor that was originally identified as an effector of Notch signaling involved in neuronal differentiation (Kageyama and Ohtsuka, 1999). Hes-1 acts as an important transcription repressor for neural stem cell differentiation by binding to N-box or preventing other activators, such as Mash1 and E47, from binding to E-box of gene promoter (Sasai et al., 1992; Kageyama et al., 2008). Overexpression of Hes- 1 in mouse embryos inhibits neurogenesis and maintains neural stem cells (Ishibashi et al., 1994). Conversely, Hes-1 knock-out mice show accelerated neurogenesis and increased expression of several proneural genes (Ishibashi et al., 1995). Yet, with the role of Hes-1 involved in neuronal differentiation well documented, its role in differentiated neurons is much less known. This issue was also examined here. In analysis of the rat GluR1 promoter, we found that it contains seven N-boxes (CACNAG or its reversed form CTNGTG) (see Fig. 1A). Therefore, we examined whether Hes-1 may regulate GluR1 expression in cultured cortical neurons.

c-Jun N-terminal kinase (JNK) is a member of the mitogenactivated protein kinase (MAPK) family that phosphorylates specific substrate proteins to regulate various cellular and physiological functions including stress-induced apoptosis (Davis, 2000; Chang and Karin, 2001). JNK activation was also found to mediate amyloid- $\beta$-induced impairment of LTP in hippocampal neurons (Costello and Herron, 2004). Moreover, JNK was found to directly phosphorylate GluR2 and GluR4 and regulate AMPA receptor traf- 
ficking in a bidirectional manner (Thomas et al., 2008). These results suggest that JNK may modulate synaptic plasticity. In this study, we aimed to examine whether JNK1 may regulate GluR1 expression through the mediation of Hes-1 and the molecular mechanism underlying this regulation. We also investigated the role of JNK1-Hes1-GluR1 signaling in regulation of AMPA-evoked calcium influx in cultured cortical neurons.

\section{Materials and Methods}

Cell culture. The procedure used for preparation of primary cortical culture from rat was adopted from that of Yang et al. (2006) with slight modifications. Briefly, embryonic primary cortical neurons were prepared from E18 to E19 of Sprague Dawley rats. The cortex from the embryo was dissociated with $100 \mathrm{U} / \mathrm{ml}$ papain and planted into 12 -well culture dishes precoated with $100 \mu \mathrm{g} / \mathrm{ml}$ poly-L-lysine at a density of $3 \times$ $10^{5}$ cells $/ \mathrm{ml}$ with minimal essential medium containing $5 \%$ calf serum, $5 \%$ horse serum, and 2\% B27-Neurobasal medium (Invitrogen). In some experiments, HEK293T cells were used. HEK293T cells were maintained in DMEM supplemented with $10 \%$ fetal calf serum. Cultures were maintained at $37^{\circ} \mathrm{C}$ in a humidified atmosphere at $5 \% \mathrm{CO}_{2}$. For transfection, primary culture in each well was incubated with $1.6 \mu \mathrm{g}$ of DNA mixed with $4 \mu \mathrm{l}$ of Lipofectamine 2000 reagent (Invitrogen) at DIV7 to DIV9 for $48 \mathrm{~h}$.

Animals. For preparation of the cytosol fraction and nuclear fraction from hippocampal tissue and cortical tissue, adult male Sprague Dawley rats $(200-250 \mathrm{~g})$ were used.

Plasmid construction, mutagenesis, and transfection. The GluR1 promoter-luciferase plasmid was constructed based on the promoter sequence of rat GluR1 gene (GenBank accession number AF302117) (Borges and Dingledine, 2001). A $2 \mathrm{~kb}$ region corresponding to -1975 to +8 was amplified by PCR using forward primer, $5^{\prime}$-CTTCACTGCAGG ATAAATGGTGG, and reverse primer, $5^{\prime}$-CAAAGATGTACGGCATAT TCC. The PCR product was subcloned between the XhoI and HindIII sites of pGL3 basic vector (Promega). For construction of the N-terminal Flag-tagged Hes1 plasmid, the full-length Hes 1 gene (GenBank accession number BC061730) was cloned by amplifying the rat hippocampal cDNA with forward primer, 5' -ATGCCAGCTGATATAATGG-3', and reverse primer, 5' -TCAGTTCCGCCACGGCCTC-3'. The PCR product was subcloned between the BamHI and EcoRI sites of pCMV-Tag 2B vector. The C-terminal Myc-tagged rat Mash1 gene (GenBank accession number NM_022384) was cloned in a similar way by using the forward primer, 5'-ATGGAGAGCTCTGGCAAGATG-3', and reverse primer, 5'-GAACCAGTTGGTAAAGTCCAGC-3'. The PCR product was subcloned between the BamHI and EcoRI sites of pCDNA4 vector. The $\mathrm{N}$-terminal Flag-tagged rat E47 gene (GenBank accession number NM_133524) was cloned by using the forward primer, ATGATGAACC AGTCTCAGAG-3', and reverse primer, TCACAGGTGCCCAGCTGG ATTG-3'. The PCR product was subcloned between the EcoRI and XholI sites of pCMV-Tag $2 \mathrm{~B}$ vector. The $\mathrm{N}$-terminal Flag-tagged full-length rat Hes5 gene (GenBank accession number NM_024383) was cloned and subcloned into the pCMV-Tag 2B vector as described previously (Lee et al., 2007). The N-terminal Flag-tagged full-length rat JNK1 $\beta 1$ plasmid was purchased from Addgene (plasmid 13752). The JNK1 plasmid was also subcloned into the $\mathrm{N}$-terminal HA-tagged pcDNA3 vector. All the vectors used are mammalian expression vectors. For mutagenesis, the N-box of the GluR1-promoter was mutated according to the method described by Takebayashi et al. (1994). Sequences of CACAAG and CTGGTG on each N-box were replaced by CCATGG and GTCGAC, respectively, by using the QuikChange Mutagenesis kit (Stratagene) and confirmed by sequencing.

Promoter-luciferase assay. Primary cortical cultures were transfected with $1.6 \mu \mathrm{g}$ of various expression plasmids. For cotransfection experiment, $0.6 \mu \mathrm{g}$ of pGL3-GluR1 promoter-firefly luciferase plasmid, $0.4 \mu \mathrm{g}$ of Flag-Hes-1 plasmid, and $0.6 \mu \mathrm{g}$ of JNK1 (or sh-JNK1) plasmid were transfected to cultured cortical neurons by using Lipofectamine 2000 (Invitrogen). For cotransfection of four plasmids, $0.4 \mu \mathrm{g}$ each of MycMash1 plasmid, Flag-E47 plasmid, His-Hes-1plasmid, and pGL3-GluR1 promoter-luciferase plasmid were transfected to cortical neurons. For siRNA transfection, 40 nM Hes-1 siRNA or control siRNA was cotrans- fected with $0.6 \mu \mathrm{g}$ of pGL3-GluR1 promoter-luciferase plasmid. In each experiment, a total of $1.6 \mu \mathrm{g}$ of DNA was transfected to cultured cortical neurons. If the amount of the transfected DNA is $<1.6 \mu \mathrm{g}$, additional Flag-vector plasmid will be transfected to reach a total of $1.6 \mu \mathrm{g}$. In addition, $7.5 \mathrm{ng}$ of CMV-promoter Renilla luciferase was transfected as an internal control. Forty-eight hours after transfection, cells were subjected to luciferase activity assay using the Dual-Glo luciferase assay system (Promega) and the TD-20/20 Luminometer (Turner Designs Hydrocarbon Instruments). The relative activity was normalized to the Renilla luciferase activity.

$\mathrm{His}_{6}$ fusion protein purification. For construction of the His-Hes-1 plasmid, full-length Hes-1 was subcloned into the pRSET-B vector. The Hes1S263A mutant plasmid was generated using the QuikChange Site-Directed Mutagenesis kit (Stratagene). Bacteria culture of Rosetta-gami 2 (DE3) pLysS (Novagen) transformed with His-Hes-1 or His-Hes-1S263A plasmids was grown at $37^{\circ} \mathrm{C}$ until it reaches $\mathrm{OD}_{600} 0.3-0.4$ before isopropyl D-thiogalactopyranoside (Sigma-Aldrich) was added to reach a final concentration of $1 \mathrm{~mm}$. After further growth at $37^{\circ} \mathrm{C}$ for $2 \mathrm{~h}$, cells were resuspended in Bugbuster protein extraction reagent (Novagen) for protein extraction according to the instruction manual. Various His-fusion proteins were purified using the nickel-nitrilotriacetic acid His-Bind resin (Novagen). The purified proteins were eluted with $0.5 \mathrm{M}$ imidazole and further dialyzed in 1 L of dialysis buffer containing $20 \mathrm{~mm}$ Tris, $\mathrm{pH}$ 8.0, 0.2 mM EDTA, $100 \mathrm{~mm}$ $\mathrm{KCl}, 20 \%$ glycerol, $0.5 \mathrm{~mm}$ DTT, and $0.2 \mathrm{~mm}$ PMSF.

In vitro kinase assay. For in vitro kinase assay, purified His-Hes-1 protein and His-Hes-1S263A mutant protein ( $1 \mu \mathrm{g}$ each $)$ were incubated with active JNK1 (40 ng) or active JNK2 (40 ng) (Cell Signaling) in a total volume of $40 \mu \mathrm{l}$ of kinase buffer $(25 \mathrm{~mm}$ Tris- $\mathrm{HCl}$ at $\mathrm{pH} 7.5,5 \mathrm{~mm}$ $\beta$-glycerophosphate, $2 \mathrm{~mm}$ DTT, $0.1 \mathrm{~mm} \mathrm{Na}_{3} \mathrm{VO}_{4}, 10 \mathrm{~mm} \mathrm{MgCl}_{2}, 0.1 \mathrm{~mm}$ ATP, and $\left.200 \mu \mathrm{Ci} / \mathrm{ml}\left[\gamma_{-}{ }^{32} \mathrm{P}\right] \mathrm{ATP}\right)$ at $30^{\circ} \mathrm{C}$ for $30 \mathrm{~min}$. The reaction was stopped by addition of SDS-containing loading buffer and heated at $95^{\circ} \mathrm{C}$ for $10 \mathrm{~min}$. The mixture $(20 \mu \mathrm{l})$ was then separated by $10 \%$ SDS-PAGE for autoradiography and Western blot.

RNA interference. The Hes-1 siRNA was designed according to that described by Ju et al. (2004) and listed by QIAGEN. The sequences for Hes-1 siRNA were as follows: 5' -CCACGTGUGAGGGCGUUAAdTdT-3' (sense) and 5'-UUAACGCCCUCACACGUGGdAdC-3' (antisense). The sequences for GluR1 siRNA were as follows: $5^{\prime}$-GCAUUAUCGACCAUUACA AdTdT-3' (sense) and 5'-UUGUAAUGGUCGAUAAUGCdTdT-3' (antisense). The Silencer Negative Control number 1 siRNA (control siRNA) was used as a control. These are the siRNAs with sequences that do not target any gene product [Applied Biosystems (ABI)]. All the siRNAs were synthesized from ABI. In addition, two sequences for JNK1 shRNA were used in this study. The sequence for sh-JNK1-1 was as follows: 5 '-CC GGCCATTTCAGAATCAGACCCATCTCGAGATGGGTCTGATTCTGAA ATGGTTTTTG-3', and the sequence for sh-JNK1-2 was as follows: 5'-CC GGGCCTAGTAATATAGTAGTCAACTCGAGTTGACTACTATATTACT AGGCTTTTTG-3'. sh-JNK1-1 and sh-JNK1-2 were cloned into the pLKO.1-shLuc vector and transfected to cultured cortical neurons by using Lipofectamine 2000. In the control group, the pLKO.1-shLuc vector was transfected. sh-JNK1-1, sh-JNK1-2, and control vector were all obtained from the National RNAi Core Facility of the Genomic Research Center (Academia Sinica, Taipei, Taiwan).

Electrophoretic mobility gel shift assay. For electrophoretic mobility gel shift assay (EMSA) experiments, the sequence of each GluR1 promoter probe containing the N-box (underlined) was as follows (" $\mathrm{m}$ " represents the mutant probe, and capital letters represent the mutation sites): GluR1-N1: $5^{\prime}$ gataaccactggtgttagggac-3'; GluR1-N1-m: 5' -gataaccaGtCgACttagggac-3'; GluR1-N2N3: 5' -aaagacacaagaaagcacaagacacctggccac-3'; GluR1-N2mN3: 5' -aaagacCATGgaaagcacaagacacctggccac- $3^{\prime}$; GluR1-N2N3m: 5' -aaagacaca agaaagcCATGgacacctggccac-3'; GluR1-N2mN3m:5'-aaagacCATGgaaagcC ATGgacacctggccac-3'; GluR1-N4: 5' -gtgctgtgctggtgaaggagt-3'; GluR1N4-m: 5' -gtgctgtgGtCgACaaggagt-3'; GluR1-N5: 5'-ctttaagtccacaagcccaga g-3'; GluR1-N5-m: 5'-ctttaagtccCATGgcccagag-3'; GluR1-N6: 5' -gtaaatcc cacaagttgtcctc- ${ }^{\prime}$; GluR1-N6-m: $5^{\prime}$-gtaaatcccCATGgttgtcctc- ${ }^{\prime}$; GluR1-N7: $5^{\prime}$-gttcaagcagctggtgaatccggggc-3'; GluR1-N7-m: $\quad 5^{\prime}$-gttcaagcag GtCgACaatccggggc- $3^{\prime}$.

The N-box-containing probes were annealed and labeled with $[\gamma$ ${ }^{32} \mathrm{P}$ ]ATP (3000 Ci/mmol) with T4-polynucleotide kinase (New England 
Biolabs). The unincorporated label was removed by using G-25 columns (GE Healthcare). Labeled probes were incubated with $1 \mu \mathrm{g}$ of His-Hes-1, which was purified from Escherichia coli at room temperature for $30 \mathrm{~min}$. For supershift experiment, anti-His antibody or control IgG was added to the reaction for an additional $30 \mathrm{~min}$. The DNA-protein mixture was analyzed by electrophoresis on non-denaturing $4.5 \%$ polyacrylamide gels run at constant $200 \mathrm{~V}$ for $2 \mathrm{~h}$ in $0.5 \times \mathrm{TBE}$. The gels were dried and exposed to $\mathrm{x}$-ray film (Eastman Kodak).

Western blot. Immunoblotting for cultured cells was performed as described previously (Yang et al., 2006). For Western blot of brain tissues, the cortex tissue and the hippocampal tissue were dissected out from the rat brain and prepared in the same way. Briefly, neurons were lysed and scraped in buffer containing $50 \mathrm{~mm}$ Tris- $\mathrm{HCl}, \mathrm{pH} 8.0,150 \mathrm{~mm}$ $\mathrm{NaCl}, 2 \mathrm{~mm}$ EDTA, $1 \mathrm{~mm} \mathrm{Na}_{3} \mathrm{VO}_{4}, 10 \mathrm{~mm} \mathrm{NaF}$, and $1 \mathrm{~mm}$ PMSF plus $2 \%$ SDS, $2 \%$ Triton X-100, $20 \mu \mathrm{g} / \mathrm{ml}$ pepstatin A, $20 \mu \mathrm{g} / \mathrm{ml}$ leupeptin, and 20 $\mu \mathrm{g} / \mathrm{ml}$ aprotinin. The lysate was centrifuged at $14,000 \times g$ for $1 \mathrm{~h}$ at $4^{\circ} \mathrm{C}$ to obtain the crude cytosolic fraction. HEK293T cells were lysed in HEPES buffer containing $150 \mathrm{~mm} \mathrm{NaCl}, 5 \mathrm{~mm}$ EDTA, $10 \mu \mathrm{g} / \mathrm{ml}$ aprotinin, $5 \mu \mathrm{g} / \mathrm{ml}$ leupeptin, $10 \%$ glycerol, and $1 \%$ Triton X-100. Equal amounts of extract were resolved by $10 \%$ SDS-PAGE and transferred to the PVDF (polyvinylidene difluoride) membrane (Millipore). The primary antibodies used were rabbit anti-Hes-1 (Millipore Bioscience Research Reagents), anti-Hes-5 (Millipore Bioscience Research Reagents), anti-GluR1 (Millipore Bioscience Research Reagents), anti-JNK1 (for Western blot; Santa Cruz; for immunohistochemistry; Abnova), antiHNRNP A1 (Acris Antibodies), anti-microtubule-associated protein 2 (MAP2) (Millipore Bioscience Research Reagents), mouse anti-Flag (M2) (Sigma-Aldrich), anti-His (Millipore), anti-phospho-JNK (Thr183, Tyr-185) (Santa Cruz) and anti-NeuN (Millipore Bioscience Research Reagents), anti-SNAP-25 (Epitomics), anti-PSD-95 (GeneTex). After incubation with the specific primary antibody, HRP-conjugated secondary antibodies were added. Membrane was developed by reacting with chemiluminescence HRP substrate and exposed to the LAS-3000 image system (Fujifilm) for visualization of protein bands.

Quantitative real-time PCR. Total RNA from primary cortical neurons was isolated using the RNAspin mini kit (GE Healthcare). Purified RNA (1 $\mu \mathrm{g}$ ) was reverse-transcripted to cDNA by SuperScript III reverse transcriptase (Invitrogen). Quantitative real-time PCR was performed using the GeneAmp 7500 Sequence Detection System with Power SYBR Green PCR reagents (Takara) according to the instruction manual (ABI). The Hes-1 primers were purchased from QIAGEN (catalog \#QTO1081094) for the purpose of SYBR Green real-time PCR. The primers for GluR1 were designed according to that of Pickering et al. (2006). The sequence for the forward primer was as follows: $5^{\prime}$-GCTTCATGGACATTGACTTA-3', and that for the reverse primer was as follows: $5^{\prime}$-ATCTCAAGTCGGTAGGAG TA-3'. Each sample was also run with the HPRT primers under identical conditions. The sequence for the forward primer was $5^{\prime}$-GCCGACCGGT TCTGTCAT- $3^{\prime}$ and that for the reverse primer was $5^{\prime}$-TCATAACCTGGT TCATCATCACTAATC-3'. The amount of Hes-1 and GluR1 gene expression is normalized to that of HPRT gene expression.

The $P 2$ fraction preparation. A frozen rat brain CA tissue was thawed at $4^{\circ} \mathrm{C}$ and homogenized by a glass homogenizer with 50 strokes in $0.3 \mathrm{ml}$ of ice-cold lysis buffer containing $320 \mathrm{~mm}$ sucrose, 4 mM HEPES, pH 7.4, 1 mM EGTA, and protease inhibitor mix (Roche). Homogenates were centrifuged at $700 \times g$ for $10 \mathrm{~min}$ at $4^{\circ} \mathrm{C}$. The supernatant (S1) was centrifuged again at $9250 \times g$ for 15 min to obtain the crude synaptosomal membrane fraction (P2) and the supernatant fraction (S2). The P2 fraction was washed with ice-cold lysis buffer and centrifuged at $10,000 \times g$ for $10 \mathrm{~min}$ at $4^{\circ} \mathrm{C}$ twice. Cultured cortical neurons were incubated with anisomycin $(100 \mathrm{ng} / \mathrm{ml})$ and/or SP600125 $(25 \mu \mathrm{g} / \mathrm{ml})$ for $18 \mathrm{~h}$ at DIV13. Neurons were scraped in $0.3 \mathrm{ml}$ of ice-cold lysis buffer as described above and were homogenized by a glass homogenizer with 50 strokes. The P2 fraction preparation for cultured cortical neurons was the same as that for hippocampal CA1 neurons.

Drugs. NMDA was purchased from Tocris. $2 \mathrm{H}$-Dibenzo[ $c d, g]$ indazol-6one (SP600125), 2-morpholin-4-yl-8-phenylchromen-4-one (LY294002), 2-(2-amino-3-methoxyphenyl)-4H-1-benzopyran-4-one (PD98059), and 4-[4-(4-fluorophenyl)-2-(4-methylsulfinylphenyl)-1 $H$-imidazol-5-yl]pyridine (SB203580) were purchased from Calbiochem. $N$-[N-(3,5-Difluoro-
phenacetyl-L-alanyl)]-S-phenylglycine $t$-butyl ester (DAPT) and TTX were purchased from Tocris Bioscience.

Calcium influx imaging. The cortical neurons were incubated with 5 $\mu \mathrm{M}$ Fluo-4 AM dye (Invitrogen) at $25^{\circ} \mathrm{C}$ for $1 \mathrm{~h}$ in dark and washed three times in $20 \mathrm{~mm}$ HEPES ( $130 \mathrm{~mm} \mathrm{NaCl}, 5.4 \mathrm{~mm} \mathrm{KCl}, 1.8 \mathrm{mM} \mathrm{CaCl}_{2}, 0.8 \mathrm{~mm}$ $\mathrm{MgCl}_{2}$, and $5.5 \mathrm{~mm}$ glucose at $\mathrm{pH} 7.4$ ) to remove extracellular Fluo-4 AM dye. Cortical neurons were subjected to laser scanning by using the UltraVIEW Live Cell confocal microscopy (PerkinElmer). Each image was scanned for $500 \mathrm{~ms}$ at $30 \mathrm{~s}$ interval, and a series of images was obtained over a total period of $10 \mathrm{~min}$ with the excitation and emission wavelengths set at 488 and $526 \mathrm{~nm}$, respectively. To suppress the activity of the voltage-sensitive $\mathrm{Na}^{+}$channels, voltage-sensitive $\mathrm{Ca}^{2+}$ channels, and NMDA receptor channels, the cultured neurons were preincubated with HBSS containing an antagonist mixture $[10 \mu \mathrm{M}$ tetrodotoxin, $50 \mu \mathrm{M}$ 2-amino-5-phosphonopentanoic acid (APV), and $100 \mu \mathrm{M}$ nifedipine]. After recording the basal fluorescence intensity for $1 \mathrm{~min}, 30 \mu \mathrm{M}$ AMPA was added to the cultured medium to stimulate the AMPA receptors. The obtained images were quantified for changes in fluorescence intensity within the cell.

Immunofluorescence staining. Cultured cortical neurons were fixed with $4 \%$ paraformaldehyde $4 \%$ sucrose for $10 \mathrm{~min}$ at room temperature and followed by permeabilization with $0.1 \%$ Triton X-100 for $20 \mathrm{~min}$ at room temperature. Primary rabbit anti-HA, anti-Hes-1, anti-GluR1 (Millipore Bioscience Research Reagents), anti-JNK1 (Tocris), and anti-PSD-95 (GeneTex) antibodies were added with $5 \%$ goat serum at $4^{\circ} \mathrm{C}$ overnight. Cy3 donkey anti-rabbit antibody (Jackson ImmunoResearch Laboratories) was incubated with these neurons for $1 \mathrm{~h}$ at room temperature.

Statistics. Data were analyzed with Student's $t$ test (for two groups) or one-way ANOVA followed by post hoc Dunnett's $t$ test (for comparisons between an experimental group and the common control group) or Newman-Keuls multiple comparisons (for comparisons between experimental groups).

\section{Results}

Hes-1 suppresses GluR1 promoter activity and GluR1 expression in primary cortical neurons

Previous study has shown that Hes-5, another transcription repressor of the bHLH family, impairs contextual fear memory formation in rats (Lee et al., 2007). This result suggests that the Hes family proteins may inhibit neuronal plasticity by suppressing the expression of downstream genes that enhance neuronal plasticity. However, GluR1 knock-out mice exhibit impaired contextual fear memory (Humeau et al., 2007) and impaired hippocampal LTP (Mack et al., 2001). Further analysis of the rat GluR1 promoter revealed that it contains seven N-boxes (N1 to N7) (CACNAG or its reverse form CTNGTG) within a $2 \mathrm{~kb}$ length sequence $(-1975$ to +8$)$ as shown in Figure $1 A$. We hypothesize that the Hes family proteins may downregulate GluR1 expression and GluR1-mediated neuronal plasticity through repressing GluR1 promoter activity. To examine whether these $\mathrm{N}$-boxes play a role in repressing GluR1 promoter activity, we have cloned $-1975 /+8$ (containing seven N-boxes) rat GluR1 promoter to pGL3 luciferase reporter vector and transfected this promoter construct (GluR1-P) to primary cortical neurons at DIV7 to DIV9. Luciferase assay was performed $48 \mathrm{~h}$ later. Results revealed that transfection of GluR1-P showed $\sim 10$-fold higher luciferase activity than the pGL3 basic control (Fig. $1 B)(p<$ 0.001 ). Because both Hes- 1 and Hes- 5 were found to suppress downstream genes through binding to N-box (Sasai et al., 1992; Kageyama et al., 2005), we next examined whether Hes-1 and Hes- 5 suppress GluR1 promoter activity through binding to the GluR1 promoter. We have cotransfected Flag-Hes-1 plasmid and GluR1 promoter reporter plasmid to primary cortical neurons. Results revealed that transfection of Flag-Hes-1 (0.4 $\mu \mathrm{g})$ dramatically decreased GluR1 promoter activity compared with transfection of pGL3-GluR1 reporter plasmid alone $(0.6 \mu \mathrm{g})(p<$ 
A

\begin{tabular}{|c|c|c|c|c|c|c|c|c|}
\hline-1975 & & & & & -13 & 735 & $+1+8$ & \\
\hline ctggtg & cacaag & cacaag & ctggtg & cacaag & cacaag & etggtg & ATG & Luciferase \\
\hline N1 & N2 & N3 & N4 & N5 & N6 & N7 & & \\
\hline
\end{tabular}

B

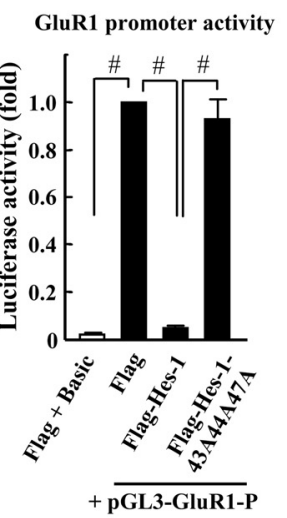

$\mathbf{F}$

Immunohistochemistry: NeuN+Hes-1
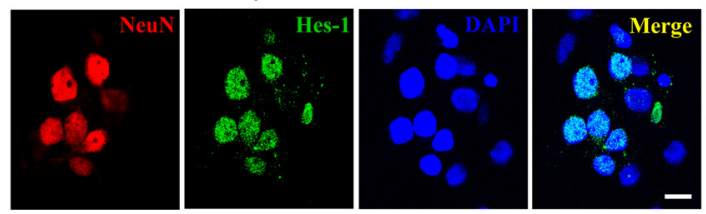

Immunohistochemistry: NeuN+GluR1
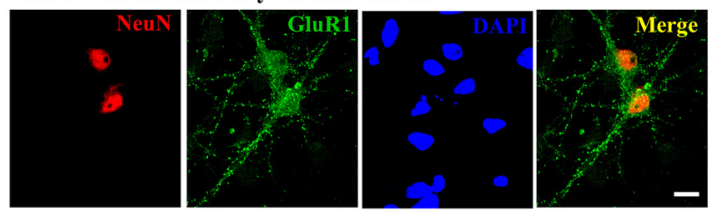

D

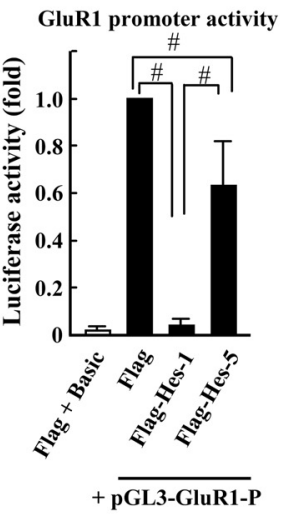

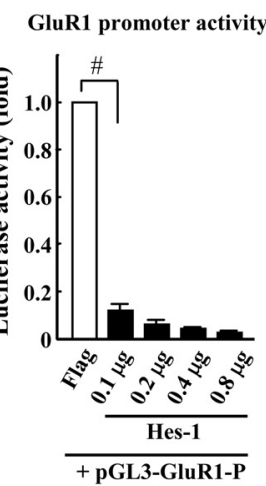

E

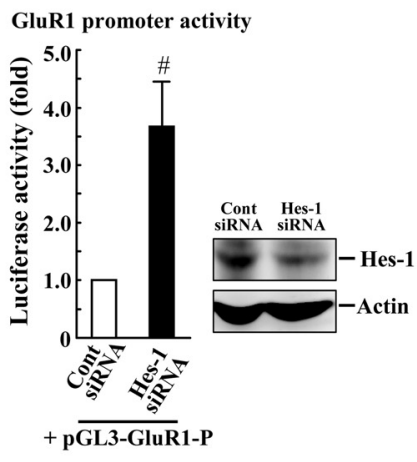

G

Adult rat brain

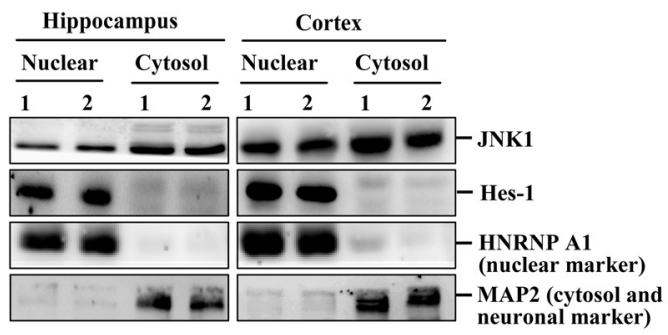

H

Co-transfection: Flag-Hes-1+GFP

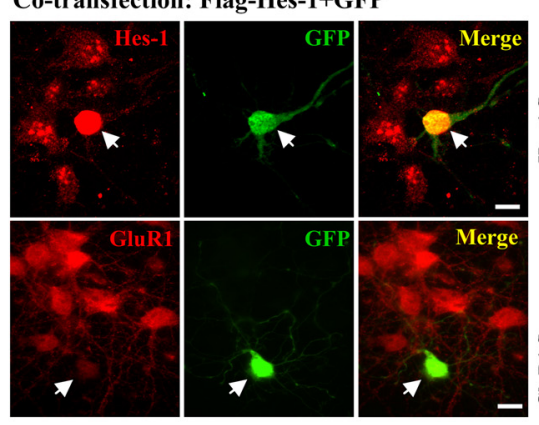

I

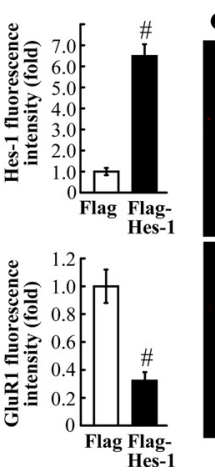

Co-transfection: Hes-1 siRNA+GFP

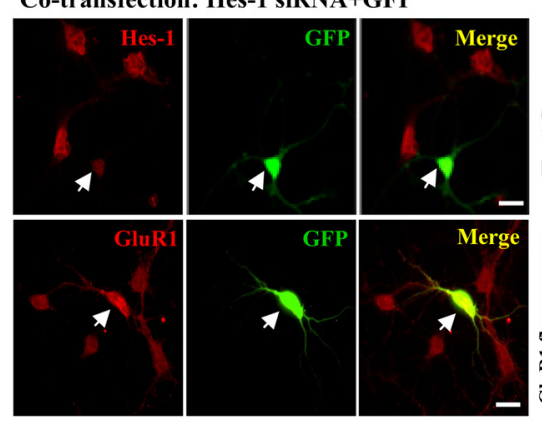

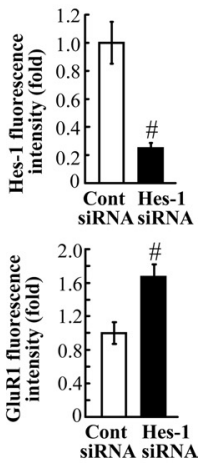

Figure 1. Hes-1 suppresses GluR1 promoter activity and GluR1 expression in primary cortical neurons. $A$, Schematic diagram of the $2 \mathrm{~kb}$ rat GluR1 genomic DNA fragment consisting seven putative $\mathrm{N}$-boxes (CACNAG or its reversed form (TNGTG). -1975 is relative to the translational start site $(+1)$. $\boldsymbol{B}$, The Flag-Hes-1WT plasmid, its dominant-negative form Hes-1-43A44A47A, or Flag vector alone, and GluR1 promoter-luciferase plasmid (pGL3-GluR1-P) were cotransfected to primary cortical neurons, and GluR1 promoter activity was determined 48 $\mathrm{h}$ later. C, The Flag-Hes-1WT plasmid, Flag-Hes-5WT plasmid or Flag vector alone (0.4 $\mu \mathrm{g}$ each), and GluR1 promoter-luciferase plasmid (pGL3-GluR1-P) $(0.6 \mu \mathrm{g})$ were cotransfected to primary cortical neurons, and GluR1 promoter activity was determined $48 \mathrm{~h}$ later. Renilla luciferase as used as an internal control. $\boldsymbol{D}$, Different doses of Hes-1WT plasmid $(0.1,0.2,0.4,0.8 \mu \mathrm{g})$ and GluR1 promoter construct were cotransfected to primary cortical neurons, and GluR1 reporter assay was performed $48 \mathrm{~h}$ later. $\boldsymbol{E}$, Hes-1 siRNA ( $40 \mathrm{~nm}$ ) or control siRNA was cotransfected with GluR1 promoter construct to primary cortical neurons and GluR1 reporter assay was performed $48 \mathrm{~h}$ later. Effectiveness of Hes-1 siRNA transfection was confirmed by Western blot. The Renilla signal was used as an internal control. Data are expressed as mean \pm SEM. Experiments are in duplicate or triplicate. $\boldsymbol{F}$, Immunohistochemistry showing the colocalization of NeuN and Hes- 1 as well as NeuN and GluR1 in primary cortical neurons. DAPI was used for nucleus staining. Scale bars: top, $15 \mu \mathrm{m}$; bottom, $20 \mu \mathrm{m}$. G. Western blot showing the nuclear and cytosol expression of JNK1 and Hes-1 in adult rat brain. $\boldsymbol{H}$, Flag-Hes-1WT plasmid and GFP plasmid were cotransfected to primary cortical neurons at DIV6 and immunofluorescence staining for Hes-1 (red), GluR1 (red), and GFP (green) was performed on DIV8. Quantified results are shown on the right. Scale bar, $10 \mu \mathrm{m}$. I, Hes-1 siRNA was cotransfected with GFP plasmid on DIV6, and immunofluorescence staining against Hes-1 (red), GluR1 (red), and GFP (green) was examined on DIV8. Quantified results are shown on the right. GFP transfection and staining was performed for identification of the transfected neuron. Scale bars: top, $20 \mu \mathrm{m}$; bottom, $10 \mu \mathrm{m}$. The number of cells ranged from 15 to 20 for each group. Data are expressed as mean \pm SEM. ${ }^{\#} p<0.001$. 
A
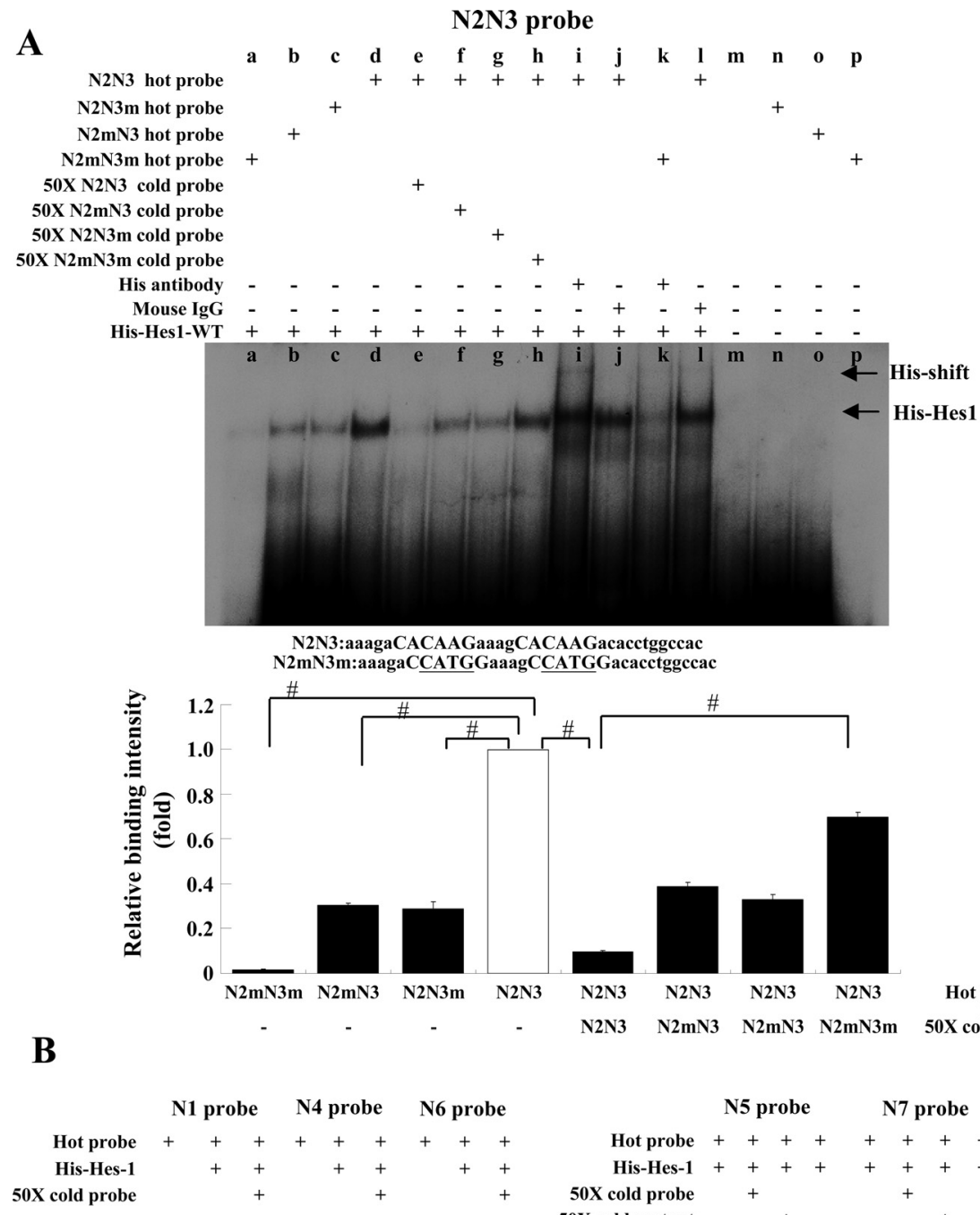

N2N3 hot probe

N2N3m hot probe

$\mathrm{N} 2 \mathrm{mN} 3$ hot probe

$\mathrm{N} 2 \mathrm{mN} 3 \mathrm{~m}$ hot probe

$50 X$ N2N3 cold probe

$50 \mathrm{X} \mathrm{N} 2 \mathrm{mN} 3$ cold probe

$50 \times$ N2N3m cold probe

50X N2mN3m cold probe

B
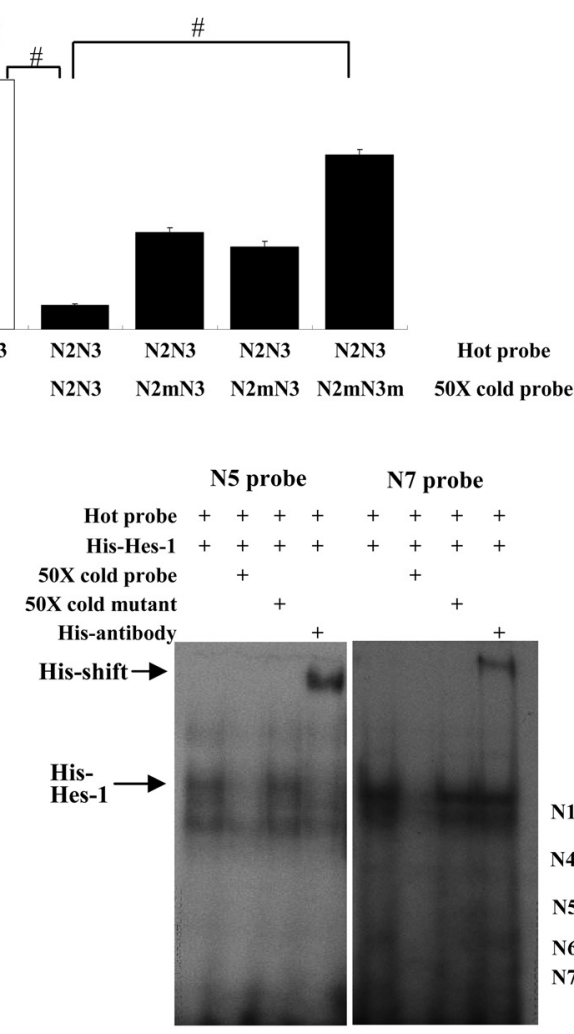

N1:gataaccaCTGGTGttagggac

N4:gtgetgtgCTGGTGaaggagt

N5:etttaagtcCACAAGcccagag

N6:gtaaatccCACAAGttgtcetc

N7:gttcaagcagCTGGTGaatecgggge

C

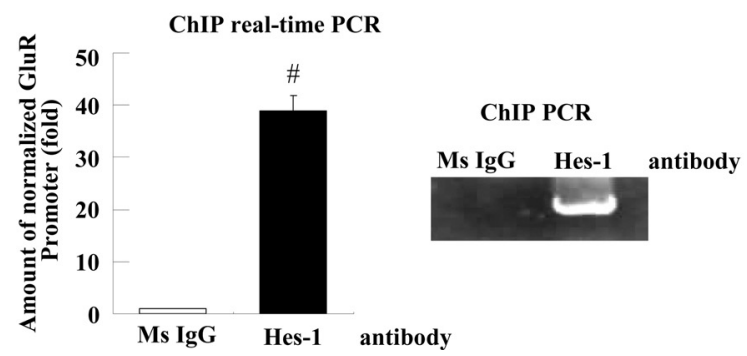

Figure 2. Hes-1 directly binds the N-boxes of the GluR1 promoter in vitro. A, EMSA assay of N2N3 probe that contains two N-boxes was labeled with $\gamma^{-32}$. Sequences for wild-type probe and mutant probe are shown at the bottom. The capital letters indicate the sequence for $\mathrm{N}$-box. The underlined capital letters represent the mutated residues. $\mathrm{m}$, Mutation. Each wild-type probe and mutant probe was incubated with His-Hes-1 that is purified from E. coli. Lane d showed that His-Hes-1 bound the N2N3 probe directly. Lanes a-c showed the binding signal of N-box double mutant N2N3 probe (N2mN3m), mutant N2 probe (N2mN3), and mutant N3 probe (N2N3m) (CACAAG $\rightarrow$ CCATGG) with His-1. Lane e showed the result with the addition of (Figure legend continues.) 
0.001). But when the pGL3-GluR1 reporter plasmid $(0.6 \mu \mathrm{g})$ was cotransfected with Flag-Hes-1-43A-44A-47A plasmid $(0.4 \mu \mathrm{g})$, the Hes-1 mutant that loses its DNA binding ability (Ström et al., 1997), GluR1 promoter activity was no longer inhibited (Fig. 1B) $(p<0.001)$. These results suggest that Hes-1 suppresses GluR1 promoter activity through binding DNA directly. In an additional experiment, we have transfected Flag-Hes-1 $(0.4 \mu \mathrm{g})$ and Flag-Hes-5 $(0.4 \mu \mathrm{g})$ to cultured cortical neurons individually. The result showed that Flag-Hes- 5 also suppressed GluR1 promoter activity, but to a much lesser extent $(p<0.001$ compared with both the Flag group and the Flag-Hes-1 group) (Fig. 1C). Thus, we have focused on Hes-1 in all future experiments. Next, we examined the dose-response effect of Flag-Hes-1 plasmid transfection on GluR1 promoter activity. Different concentrations of Flag-Hes- 1 plasmid $(0.1,0.2,0.4$, and $0.8 \mu \mathrm{g})$ and pGL3 GluR1 reported plasmid $(0.6 \mu \mathrm{g})$ were cotransfected to cultured cortical neurons. Results revealed a dose-dependent suppression of GluR1 promoter activity by Flag-Hes-1 transfection, and a concentration of Flag-Hes- 1 as low as $0.1 \mu \mathrm{g}$ strongly suppressed GluR1 promoter activity (Fig. $1 D)(p<0.001$ for all concentrations of the Hes-1 plasmid). This result suggests that Hes-1 is a dominant regulator of GluR1 promoter activity. Next, we transfected Hes-1 siRNA (40 nM) together with pGL3-GluR1 promoter plasmid $(0.6 \mu \mathrm{g})$ to cultured cortical neurons and have found that Hes-1 siRNA increased GluR1 promoter activity for 3.7-fold when compared with the control siRNA (Fig. 1E) $(p<$ 0.001 ). The effect of Hes-1 siRNA transfection in knocking down Hes-1 expression was confirmed by Western blot (Fig. $1 E$ ). This result further confirms that Hes-1 strongly and negatively regulates GluR1 promoter activity.

Next, we characterized the expression of Hes-1 and GluR1 in primary cortical neurons by immunofluorescence staining. Results revealed that both Hes-1 and GluR1 are expressed in neurons specifically, as confirmed by NeuN staining (Fig. $1 F$ ). Further Western blot analysis revealed that Hes-1 is specifically expressed in the nuclear fraction of adult cortical and hippocampal neurons (Fig. 1G).

In the following experiments, we examined whether Hes-1 also regulates GluR1 protein expression. Flag-Hes-1 plasmid ( 0.8 $\mu \mathrm{g})$ and GFP construct $(0.8 \mu \mathrm{g})$ were cotransfected to cultured cortical neurons, and immunofluorescence staining for Hes-1 and GluR1 was performed $48 \mathrm{~h}$ later. Results revealed that the fluorescence intensity for Hes-1 is apparently much higher in a cell successfully transfected with Flag-Hes-1 compared with that

\footnotetext{
$\leftarrow$

(Figure legend continued.) $50 \times$ cold N2N3 competitor probe. Lanes $f-h$ revealed that addition of $50 \times$ mutant cold competitor ( $22 \mathrm{mN} 3, \mathrm{~N} 2 \mathrm{~N} 3 \mathrm{~m}$, or $\mathrm{N} 2 \mathrm{mN} 3 \mathrm{~m}$ ) partially decreased the binding signal, but cold $\mathrm{N} 2 \mathrm{mN} 3 \mathrm{~m}$ probe cannot abolish the signal from the hot N2N3 probe. His antibody caused supershift of the band in lane i (indicated by the top arrow), but control lgG had no such an effect (lane j). Lanes $m-p$ showed the result of probe only. The binding signal for the His-Hes-1-probe complex is also shown (indicated by the bottom arrow). The capital letters at the bottom indicate the sequences for individual $\mathrm{N}$-boxes. The quantified result of binding is shown at the bottom panel. $\boldsymbol{B}$, EMSA assay of different N-probes that contain individual N-box were labeled with $\gamma^{-}{ }^{32} \mathrm{P}$. Sequences for each probe are shown at the bottom. The capital letters indicate the sequence for the N-box. Different N-probes were incubated with His-Hes- 1 that is purified from E. coli. EMSA profile revealed that Hes- 1 binds to all these N-boxes, and this binding was reversed by individual cold probe competition. The binding was further reversed by mutant cold probe competition. His antibody caused a supershift of the band (indicated by the top arrow). The binding signal for the His-Hes-1-probe complex is also shown (indicated by the bottom arrow). C, Results from ChIP assay followed by real-time PCR showing specific binding of endogenous Hes-1 to endogenous GluR1 promoter in cultured cortical neurons using the Hes-1 antibody and the primer for GluR1 promoter. Experiments are in duplicate ( $n=3$ for each group). Data are expressed as mean \pm SEM. ${ }^{\#} p<0.001$.
}

of the neighboring GFP-negative cells (Fig. $1 H$, top left). However, in a cell successfully transfected with Flag-Hes-1, the fluorescence intensity for GluR1 is much lower than that of the neighboring GFP-negative cells (Fig. $1 H$, bottom left). The quantified results are shown on the right $(p<0.001$ for both Hes-1 and GluR1). We next examined whether Hes-1 siRNA produces an opposite effect. Primary cortical neurons were cotransfected with Hes-1 siRNA (40 nm) and GFP construct $(0.8 \mu \mathrm{g})$, and immunofluorescence staining for Hes-1 and GluR1 was performed. Results revealed that a cell successfully transfected with Hes-1 siRNA showed reduced fluorescence intensity for Hes-1 compared with that of the neighboring GFP-negative cells (Fig. $1 I$, top left). But for a cell successfully transfected with Hes-1 siRNA, the fluorescence intensity for GluR1 is higher than that of the neighboring GFP-negative cells (Fig. $1 I$, bottom left). The quantified results are shown on the right $(p<0.001$ for both Hes-1 and GluR1). These results together suggest that Hes-1 negatively regulates GluR1 protein expression in cortical neurons.

\section{DNA binding analysis for Hes-1 binding to GluR1 promoter}

Hes-1 was reported to inhibit the promoter activity of target genes by directly binding to the N-box. For example, Hes-1 directly binds to the N-box on MAP2 promoter and inhibits its promoter activity in PC12 cells (Bhat et al., 2006). Hes-1 was also found to negatively autoregulate Hes-1 promoter activity through binding to three N-boxes on its own promoter (Takebayashi et al., 1994). In this experiment, we examined whether Hes- 1 binds to the N-boxes on GluR1 promoter directly using EMSA. The His-Hes-1 fusion protein was expressed and purified from $E$. coli and mixed with various $\gamma-{ }^{32} \mathrm{P}$-labeled $\mathrm{N}$-box probes. Results revealed that His-Hes-1 bound the N2N3 probe in which $\mathrm{N} 2$ and $\mathrm{N} 3$ coexist in a 33 bp length probe (lane d), and it did not bind the $\mathrm{N} 2 \mathrm{mN} 3 \mathrm{~m}$ double mutant probe (lane a) (Fig. $2 \mathrm{~A}$ ). The binding intensity was diminished for either single N-box mutant probe (N2mN3 or N2N3m) (Fig. $2 A$, lanes b and c) compared with that in lane $\mathrm{d}$. The binding affinity was verified by adding $50 \times$ cold probe as the competitor. Results showed that addition of the N2N3 cold probe completely eliminated the binding signal for His-Hes-1 (Fig. $2 \mathrm{~A}$, lanes e and d), but addition of the mutant $\mathrm{N} 2 \mathrm{mN} 3 \mathrm{~m}$ cold probe did not affect the binding ability of $\mathrm{N} 2 \mathrm{~N} 3$ hot probe (Fig. $2 \mathrm{~A}$, lanes $\mathrm{d}$ and $\mathrm{h}$ ). Figure $2 \mathrm{~A}$, lane i, showed the His antibody shift band, indicating the formation of the His-Hes1 -probe complex. These results suggest that Hes- 1 could bind to the N2- and N3-boxes of the GluR1 promoter directly. The quantified result of binding intensity is shown at the bottom of Figure $2 A$ ( $p<0.001$ for various comparisons). In addition, we found that Hes- 1 also binds to other N-boxes of the $2 \mathrm{~kb}$ GluR1 promoter and the results are shown in Figure $2 B$.

To know whether the endogenous Hes-1 protein binds to the endogenous GluR1 promoter in neurons, we have performed chromatin immunoprecipitation (ChIP) assay in cultured cortical neurons using Hes-1 antibody to immunoprecipitate the cell lysate followed by real-time PCR. Results revealed that the endogenous Hes-1 binds to GluR1 promoter in cortical neurons and this binding is confirmed by ChIP PCR and ChIP real-time PCR by using specific primer for the GluR1 promoter (Fig. 2C).

\section{Hes-1 suppresses GluR1 promoter activity through binding to $\mathrm{N}$-boxes and through preventing the formation of the Mash1/ E47 heterodimer}

The above results demonstrate that Hes-1 directly binds to all seven N-boxes on GluR1 promoter. In this experiment, we examined whether Hes- 1 binding to these N-boxes actually suppresses GluR1 
A

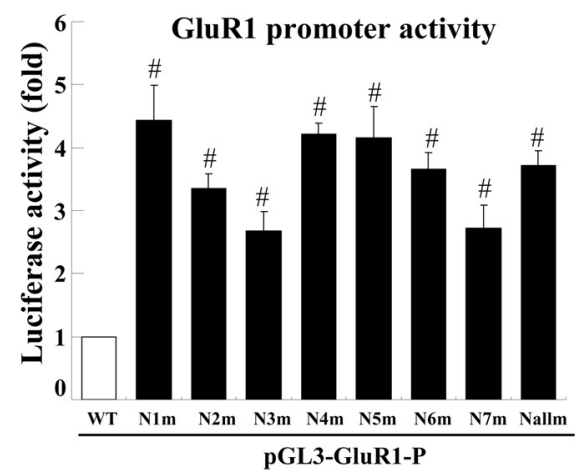

B

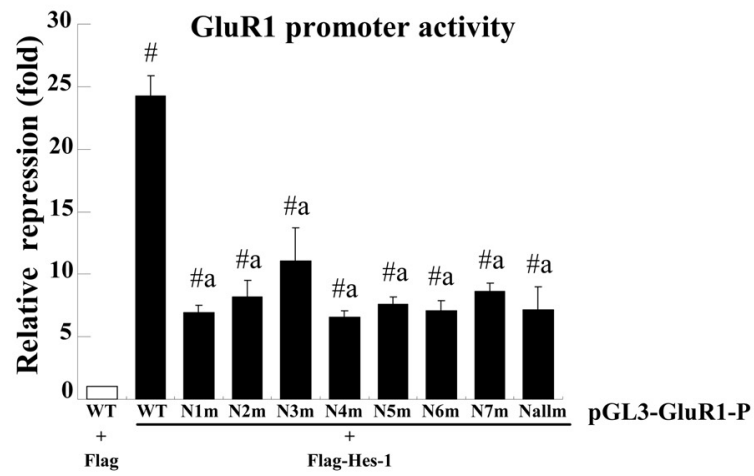

C

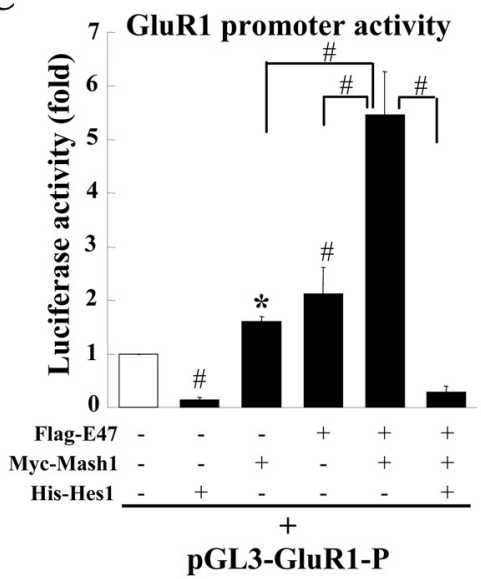

D

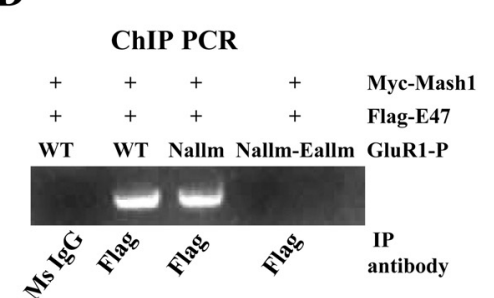

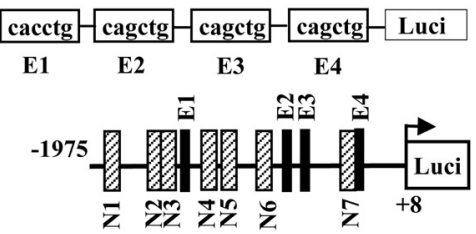

E

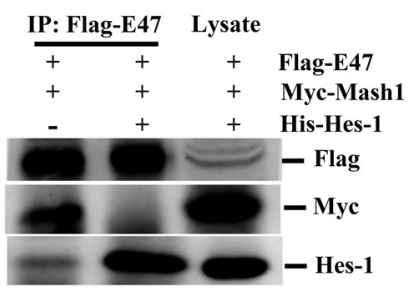

promoter activity. Wild-type pGL3-GluR1 promoter plasmid or various GluR1 promoter mutant plasmids (with each N-box been mutated individually) $(0.6 \mu \mathrm{g})$ were first transfected to cultured cortical neurons for luciferase assay. Results revealed that mutation of any single N-box (N1 to N7) of the GluR1 promoter significantly increased GluR1 promoter activity (2.7- to 4.5 -fold) when compared with the wild-type control ( $p<0.001$ for N1 to N7 mutations when compared with the wild-type group). But mutation of all seven N-boxes (Nallm group) did not produce a further enhancing effect (Fig. 3A). This result suggests that each N-box plays an important, and perhaps sufficient, role in suppressing GluR1 promoter activity. Next, we examined whether Hes-1 suppresses GluR1 promoter activity through binding to these $\mathrm{N}$-boxes and whether each N-box contributes equally to the suppressing effect of Hes-1. Flag-Hes-1 plasmid or Flagvector plasmid $(0.4 \mu \mathrm{g})$ was cotransfected with the wild-type pGL3-GluR1 promoter plasmid or various GluR1 promoter mutant plasmids with mutation on each N-box individually $(0.6 \mu \mathrm{g})$ to cultured cortical neurons for GluR1 promoter activity determination. Results revealed that transfection of Flag-Hes-1 plasmid dramatically suppressed GluR1 promoter activity for $\sim 24$-fold $(p<0.001)$. But transfection of Flag-Hes-1 with any N-box-mutated-GluR1 promoter plasmid effectively reduced the suppressing effect of Flag-Hes-1 on GluR1 promoter activity to $\sim 5$ - to 10-fold ( $p<0.001$ for N1 to N7 mutations when compared with the wildtype+Flag-Hes-1 group). (Fig. 3B). These results suggest that Hes-1 suppressed GluR1 promoter activity through binding to these $\mathrm{N}$-boxes and each N-box contributes similarly to this effect of Hes-1. In addition,

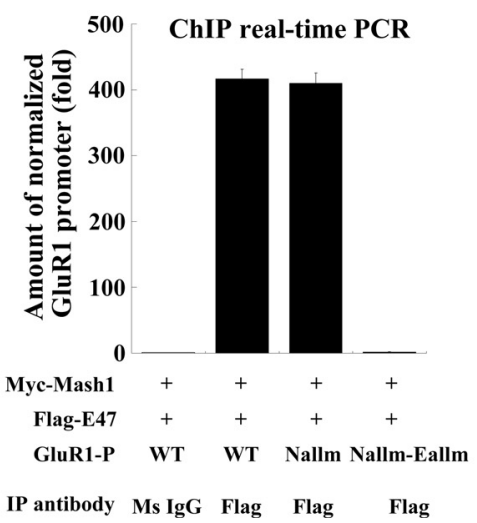

Figure 3. Hes-1 suppresses GluR1 promoter activity through binding to N-boxes and preventing Mash1/E47 from binding to E-boxes of the GluR1 promoter. $\boldsymbol{A}$, Wild-type GluR1 promoter construct or various GluR1 promoter mutant constructs with each $\mathrm{N}$-box mutated individually was transfected to primary cortical neurons, and GluR1 reporter assay was determined $48 \mathrm{~h}$ later. Nallm means all seven N-boxes were mutated. B, Flag-Hes-1WT plasmid (or Flag-vector) was cotransfected with GluR1WT promoter construct or various GluR1 promoter construct with mutation at each N-box to primary cortical neurons. GluR1 reporter assay was

\begin{abstract}
$\leftarrow$
determined $48 \mathrm{~h}$ later. C, Various combinations of the HisHes-1 plasmid, Flag-E47 plasmid, and Myc-Mash1 plasmid were cotransfected to cultured cortical neurons, and GluR1 promoter activity was determined $48 \mathrm{~h}$ later. The top right panel shows the relative position of each N-box and E-box on GluR1 promoter. D, The Myc-Mahs1, Flag-E47, and different GluR1 promoter plasmids were cotransfected to HEK293 cells followed by IP using the Flag antibody (or mouse $\lg$ G). The samples were then subjected to ChIP PCR and ChIP real-time $P C R$ to show the direct binding of E47 to the E-box of GluR1 promoter. Nallm, All N-box mutated; Nallm-Eallm, all N-box and all E-box mutated. $\boldsymbol{E}$, The same plasmids used in $\boldsymbol{C}$ were cotransfected to HEK293 cells for IP and IB experiments to show the association of E47 and Mash1, and prevention of this association by Hes-1. GluR1-P, GluR1 promoter. Experiments are in duplicate ( $n=3$ for each group). Data are expressed as mean \pm SEM. ${ }^{*} p<0.05$ and ${ }^{\#} p<0.001 ;{ }^{a}$ compared with the Flag-Hes-1WT group.
\end{abstract}


cotransfection of Flag-Hes-1 with all N-box mutant GluR1 promoter plasmid did not produce a further decreasing effect (Fig. 3B). This result suggests that mechanisms other than binding to N-boxes may also play a role in mediating the suppressing effect of Flag-Hes-1 on GluR1 promoter activity.

Hes-1 was found to downregulate target gene expression by preventing other bHLH factors, such as the Mash1/E47 heterodimer, from binding to the E-box (CANNTG) of the target gene (Sasai et al., 1992). In analyzing the $2 \mathrm{~kb}$ rat GluR1 promoter, we found that there are four E-boxes and three of them (E1, E3, and E4) are conserved in rat and mouse (Fig. 3C, top panel). Mash1 is an important bHLH activator and Mash1, together with its cofactor E47, binding to E-box promotes neuronal differentiation (Ross et al., 2003). Therefore, we also examined whether Hes-1 may suppress GluR1 promoter activity through preventing Mash1/E47 from binding to the E-box on GluR1 promoter. The pGL3-GluR1 promoter plasmid was cotransfected with various combinations of Myc-Mash1, Flag-E47, and HisHes-1 plasmids ( $0.4 \mu \mathrm{g}$ each) to cultured cortical neurons and GluR1 promoter activity was determined. Results revealed that transfection of His-Hes-1 decreased GluR1 promoter activity for $\sim 90 \%$ ( $p<0.001)$. Transfection of Myc-Mash1 increased GluR1 promoter activity for $\sim 1.6$-fold $(p<0.05)$ and transfection of Flag-E47 increased GluR1 promoter activity for $\sim 2.1$-fold $(p<$ 0.001). Cotransfection of Myc-Mash1 and Flag-E47 produced a synergistic effect in enhancing GluR1 promoter activity (for approximately fivefold; $p<0.001$ compared with the MycMash1 group and Flag-E47 group alone), but this effect was completely blocked by cotransfection with His-Hes-1 $(p<$ 0.001 compared with the Myc-Mash1 plus Flag-E47 cotransfection group) (Fig. 3C).

Next, we examined whether the Mash1/E47 complex directly binds to the E-box of GluR1 promoter. The Myc-Mash1 and Flag-E47 plasmids together with different GluR1 promoter plasmids (WT, all N-box-mutated and all N-box- and all E-boxmutated) were cotransfected to HEK293 cells. The cell lysate was immunoprecipitated with the Flag antibody and subjected to ChIP PCR using the GluR1 promoter primers. Results from both ChIP PCR and ChIP real-time PCR revealed that E47 directly binds to the E-box, but not N-box, of the GluR1 promoter (Fig. $3 D$ ). Further coimmunoprecipitation (co-IP) experiment revealed that E47 is associated with Mash1, but transfection of HisHes-1 disrupted this association (Fig. 3E). These results together suggest that Hes-1 strongly and negatively regulates GluR1 promoter activity through directly binding to the N-boxes and through preventing the association of Mash1 and E47, and preventing the Mash1/E47 complex from binding to the E-boxes on GluR1 promoter. These results also explain the residual repression observed in all N-box-mutated group (Nallm) in Figure $3 B$.

\section{Expression of Hes-1 and GluR1 is regulated by JNK signaling but not Notch signaling in primary cortical neurons}

Hes- 1 is an effector of Notch signaling that controls stem cell differentiation (Kageyama et al., 2005). When Notch is activated by its ligands expressed in neighboring cells, the intracellular domain of Notch (NICD) is cleaved by $\gamma$-secretase and translocated to the nucleus to form complex with RBP-J $\kappa$ to induce Hes-1 expression (Kageyama and Ohtsuka, 1999). In this series of experiments, we first investigated whether Notch upregulates Hes-1 expression in differentiated cortical neurons. The $\gamma$-secretase inhibitor, DAPT ( 1 and $10 \mu \mathrm{M}$ ), was added to the culture medium, and the expression level of NICD, Hes-1, and GluR1 was examined by Western blot $48 \mathrm{~h}$ later. Results revealed that DAPT ef- fectively inhibited the expression of NICD, but it did not alter the expression of Hes-1 and GluR1 (Fig. 4A). This result suggests that Hes- 1 and GluR1 expression is not regulated by Notch signaling in cortical neurons. We then studied the possible upstream molecule that regulates Hes-1 and GluR1. The inhibitors for various protein kinases were added to the culture medium, and Hes-1 expression was examined $18 \mathrm{~h}$ later. These inhibitors include LY294002 (50 $\mu \mathrm{M}$; PI3-K inhibitor), PD98059 (50 $\mu \mathrm{M}$; ERK/ MAPK inhibitor), SB203580 (20 $\mu \mathrm{M}$; p38 MAPK inhibitor), and SP600125 (25 $\mu \mathrm{M}$; JNK inhibitor). DAPT (10 $\mu \mathrm{M})$ was used as a negative control. Results revealed that SP600125 is the only inhibitor that affects Hes- 1 expression $(p<0.001$ compared with the DMSO control group) (Fig. $4 B$ ). Further analysis indicated that SP600125 decreased the expression of Hes-1, but increased the expression of GluR1 $(p<0.01)$ (Fig. $4 B$, bottom right panel). Together with our previous finding that Hes-1 siRNA transfection enhanced the fluorescence intensity of GluR1 in cultured cortical neurons (Fig. $1 \mathrm{H}$ ), these results suggest that JNK signaling may downregulate GluR1 expression through enhanced expression of Hes-1.

We next examined whether JNK signaling regulates GluR1 transcription. Anisomycin (100 ng/ml), used as a JNK activator (Iordanov et al., 1997; Fosbrink et al., 2010), was added to cultured cortical neurons, and GluR1 mRNA level was determined at different time points by real-time PCR. Results revealed that anisomycin decreased GluR1 mRNA level in a time-dependent manner, with the 4 and $18 \mathrm{~h}$ time points showing the most apparent effect $(p<0.01)$, but this effect was similarly blocked by coadministration of SP600125 ( $p<0.001$ compared with the corresponding anisomycin-alone group) (Fig. $4 C$ ). We also examined the time course effect of anisomycin on Hes-1 and GluR1 protein expression. Meanwhile, its effect on JNK activation was also determined. Results revealed that anisomycin decreased GluR1 protein level at $18 \mathrm{~h}$ later, but not at other time points $(p<0.001)$. Anisomycin also increased Hes-1 protein level in a timedependent manner starting at $2 \mathrm{~h}$ and lasted up to $18 \mathrm{~h}(p<$ $0.001)$. The effect of anisomycin on JNK activation was confirmed by an apparent increase in the level of JNK phosphorylation at Thr-183 and Thr-185. All of these effects were blocked by coadministration of the JNK inhibitor SP600125 $(p<0.001$ compared with the corresponding anisomycin group) (Fig. 4D). Although the effect of anisomycin on JNK activation lasted for $<4 \mathrm{~h}$, this time period is long enough for a kinase to transduce its downstream signaling.

In a separate experiment, we have used another JNK activator $\mathrm{H}_{2} \mathrm{O}_{2}$ to address the same issue. Results revealed that $\mathrm{H}_{2} \mathrm{O}_{2}(100$ $\mu \mathrm{M})$ treatment produced a similar effect on Hes-1 and GluR1 expression to that of anisomycin ( $p<0.01$ for Hes- 1 at 2, 4, and $18 \mathrm{~h}$, and $p<0.001$ for GluR1 at $18 \mathrm{~h}$ ), and these effects were also blocked by SP600125 cotreatment ( $p<0.001$ compared with the corresponding $\mathrm{H}_{2} \mathrm{O}_{2}$ group at 4 or $18 \mathrm{~h}$ ) (Fig. $4 E$ ). These results together indicated that JNK activation decreased GluR1 mRNA and protein expression associated with increased Hes-1 expression.

\section{JNK1 decreases GluR1 promoter activity and GluR1 expression through the mediation of Hes-1 in cultured cortical neurons}

The above results demonstrated that activation of JNK signaling decreased GluR1 expression associated with increased Hes-1 expression. We also examined whether JNK suppresses GluR1 promoter activity and whether this is mediated through Hes-1. The HA-JNK1 plasmid or two sh-JNK1 plasmids, alone or together 
A

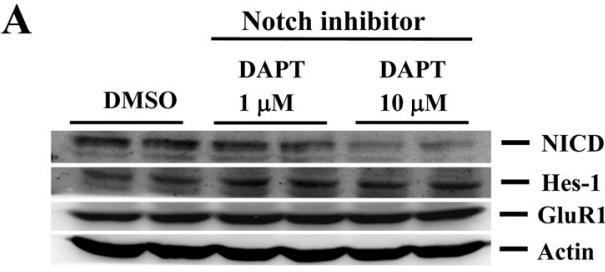

C

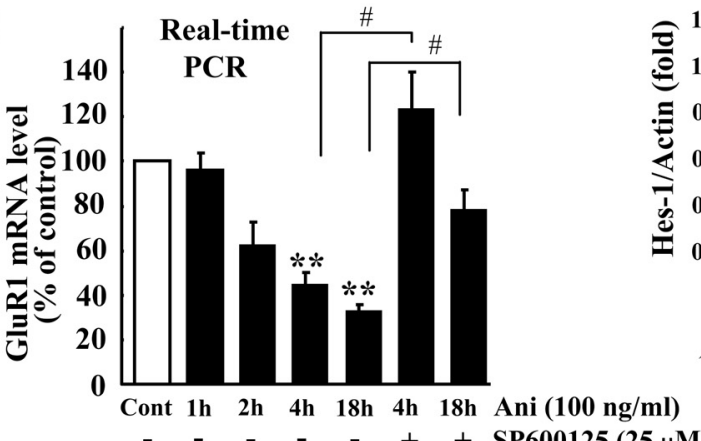

D

Cont 1h 2h 4h $18 \mathrm{~h}$ 4h $18 \mathrm{~h}$ Cont Ani $(100 \mathrm{ng} / \mathrm{ml})$

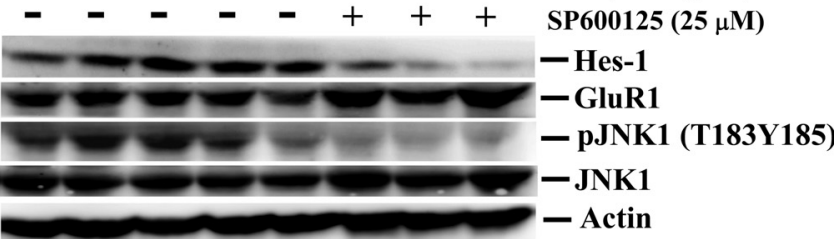

$\mathbf{E}$

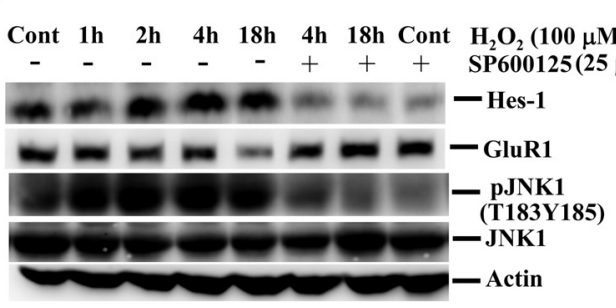

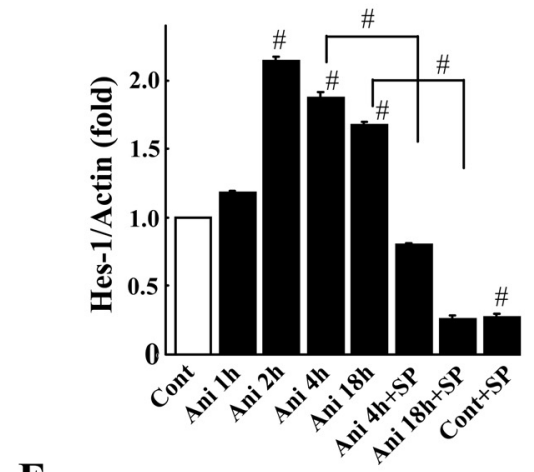

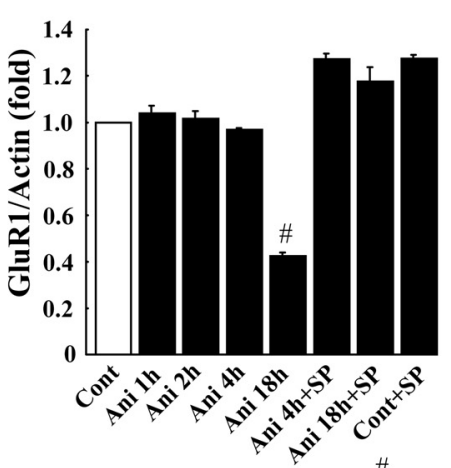

B Inhibitors

DMSO DAPT LY294002 PD98059 SB203580 SP600125
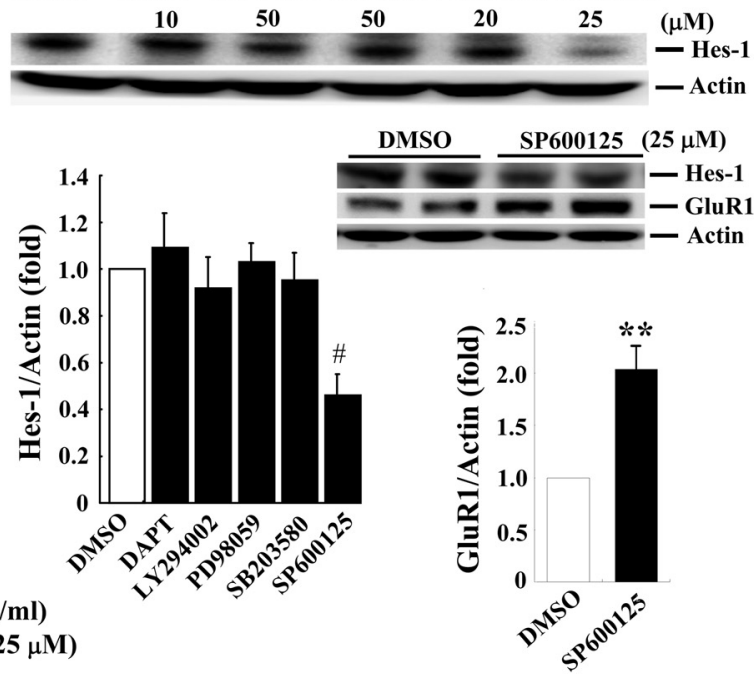
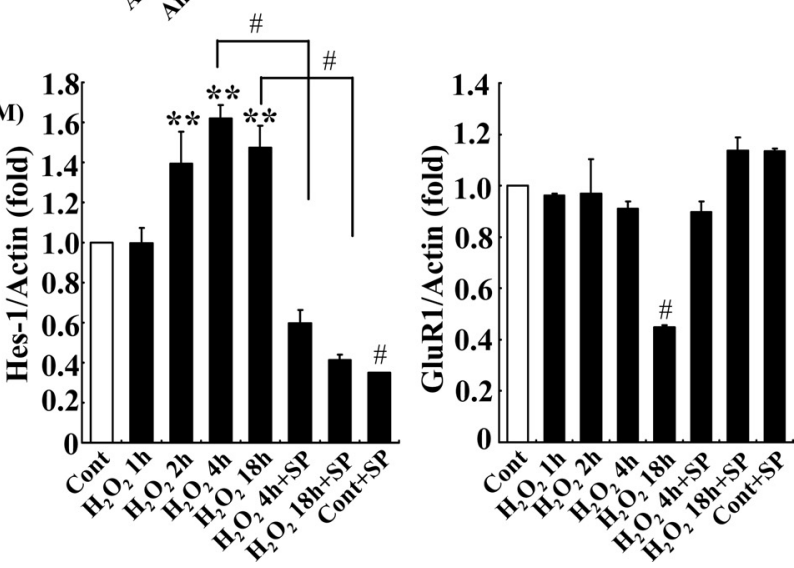

Figure 4. Hes-1 expression and GluR1 expression are regulated by JNK signaling but not Notch signaling. $A$, Notch inhibitor (DAPT) was added to cultured cortical neurons at two concentrations, and its effect on Hes-1 and GluR1 expression was examined by Western blot. Effectiveness of DAPT treatment was confirmed by Western blot against NICD. B, Specific inhibitors against Notch (DAPT), PI3-K (LY294002), ERK/MAPK (PD98059), p38 MAPK (SB203580), and JNK (SP600125) were applied to cultured cortical neurons, and their effects on Hes-1 expression were examined $24 \mathrm{~h} \mathrm{later} \mathrm{by}$ Western blot. The quantified result is shown at the bottom. Effect of the JNK inhibitor on GluR1 expression was further examined. C, Anisomycin at $100 \mathrm{ng} / \mathrm{ml}$ (to activate JNK), alone or together with JNK inhibitor, was applied to cultured cortical neurons, and GluR1 mRNA level was determined by real-time PCR at different time points. D, Anisomycin (100 ng/ml), alone or together with JNK inhibitor (SP600125), was applied to cultured cortical neurons, and their effects on the expression of Hes-1, GluR1, JNK1, and phospho-JNK1 were determined by Western blot. The quantified results are shown at the bottom. E, Time course effect of $\mathrm{H}_{2} \mathrm{O}_{2}$ and $\mathrm{SP} 600125$ on the expression of Hes-1, GluR1, JNK1, and phospho-JNK1 were determined by Western blot. The quantified results are shown on the right. Ani, Anisomycin; SP, SP600125. Experiments are in duplicate ( $n=3$ for each group). Data are expressed as mean \pm SEM. ${ }^{* *} p<0.01$ and ${ }^{\#} p<0.001$. 
A

\subsection{GluR1 promoter activity}

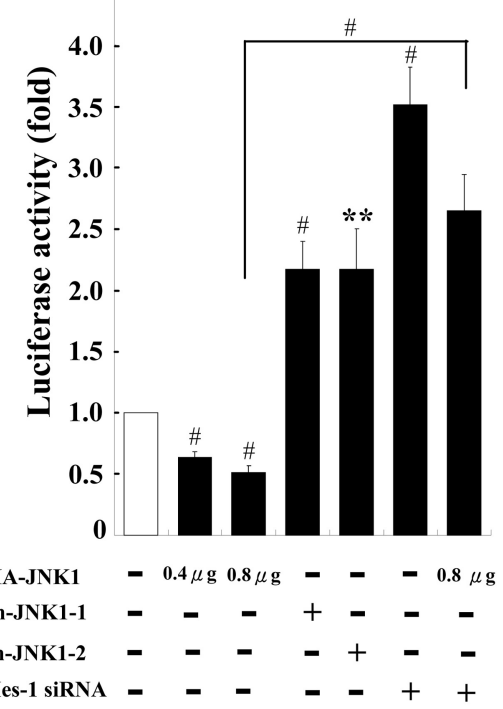

B
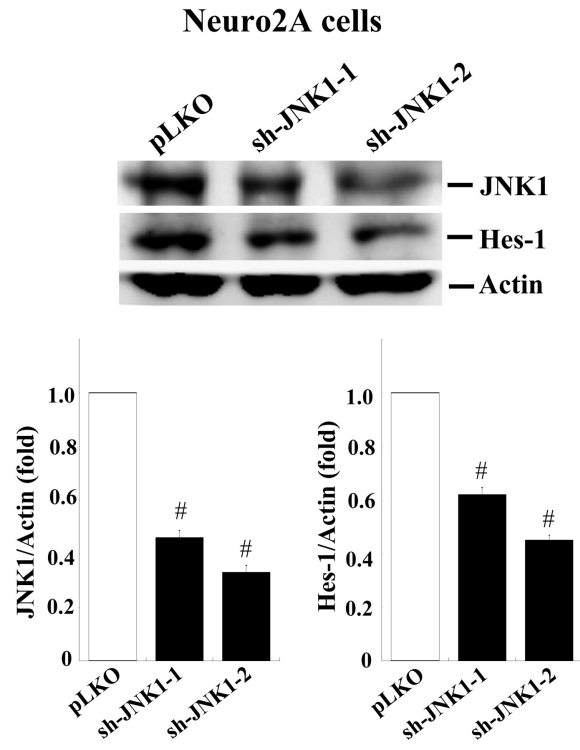

Figure 5. Overexpression of JNK1 suppressed GluR1 promoter activity that is blocked by Hes-1 siRNA cotransfection. A, HA-JNK1WT plasmid, two sh-JNK1 plasmids, and/or Hes-1 siRNA were transfected to cultured cortical neurons, and their effects on GluR1 promoter activity was determined $24 \mathrm{~h}$ later. $\boldsymbol{B}$, The effectiveness of both sh-JNK1 in knocking down JNK1 expression and their effects on Hes-1 expression were shown in Neuro2A cells. The results are also quantified. Experiments are in duplicate ( $n=3$ for each group). Data are expressed as mean \pm SEM. ${ }^{* *} p<0.01$ and ${ }^{\#} p<0.001$.

with Hes-1 siRNA, were transfected to cultured cortical neurons, and their effects on GluR1 promoter activity were determined $24 \mathrm{~h}$ later. Results revealed that JNK1 decreased GluR1 promoter activity, whereas both sh-JNK1-1 and sh-JNK1-2 increased GluR1 promoter activity $(p<0.01$ or $p<0.001)$. Transfection of Hes-1 siRNA significantly increased GluR1 promoter activity $(p<0.001)$. But the decreasing effect of JNK1 on GluR1 promoter activity was completely blocked by Hes- 1 siRNA cotransfection ( $p<0.001$ compared with the HA-JNK1 group) (Fig. $5 A)$. The effectiveness of both sh-JNK1 transfection in knocking down JNK1 expression and reducing Hes-1 expression is confirmed in Neuro2A cells $(p<0.001)$ (Fig. $5 B)$.

Next, we examined whether JNK1 decreases GluR1 protein expression in cortical neurons. Here, we used JNK overexpres- sion instead of anisomycin to activate JNK because, by using overexpression, we could identify individual neurons clearly and quantify the fluorescence intensity whereas anisomycin would cause a massive elevation in fluorescence intensity in all cells. HA-JNK1 plasmid $(0.8 \mu \mathrm{g})$ and GFP construct $(0.8 \mu \mathrm{g})$ were cotransfected to cultured cortical neurons and immunofluorescence staining for JNK1 and GluR1 was performed $48 \mathrm{~h}$ later. Results revealed that the fluorescence intensity for JNK1 is apparently higher for a cell successfully transfected with HA-JNK1 compared with that of the neighboring GFP-negative cells (Fig. $6 A$, top left). But for a cell successfully transfected with HAJNK1, the fluorescence intensity for GluR1 is lower than that of the neighboring GFP-negative cells (Fig. 6A, bottom left). The quantified result is shown on the right $(p<0.001)$.

Next, we examined whether Hes-1 siRNA blocks the suppressing effect of JNK1 on GluR1 expression. HA-JNK1 plasmid ( 0.8 $\mu \mathrm{g})$, GFP construct $(0.8 \mu \mathrm{g}$ ), and $40 \mathrm{~nm} \mathrm{Hes}-1$ siRNA (or control siRNA) were cotransfected to cultured cortical neurons, and immunofluorescence staining for GluR1 was performed $48 \mathrm{~h}$ later. Results revealed that, for a cell successfully transfected with HAJNK1 and Hes-1 siRNA (Fig. 6B, bottom left), the fluorescence intensity for GluR1 is apparently higher than that of a cell transfected with HA-JNK1 and control siRNA (Fig. $6 \mathrm{~B}$, top left) under the circumstance that the fluorescence intensity for GluR1 in non-GFP positive cells are similar in both panels. The quantified result is shown on the right $(p<0.001$ for comparison between JNK1 group with control group and $p<0.001$ for comparison between JNK1 plus Hes-1 siRNA group with control group). This result suggests that the suppressing effect of JNK1 on GluR1 expression is blocked by Hes-1 siRNA cotransfection.

In addition, we examined whether both sh-JNK1 plasmids decreased GluR1 expression. sh-JNK1-1 $(0.8 \mu \mathrm{g})$ and the GFP construct $(0.8 \mu \mathrm{g})$ were cotransfected to cultured cortical neurons, and immunofluorescence staining for JNK1 and GluR1 was performed $48 \mathrm{~h}$ later. Results revealed that, for a cell successfully transfected with sh-JNK1-1, the fluorescence intensity for JNK-1 is much lower compared with that of the neighboring GFPnegative cells (Fig. 6C, top left). However, the fluorescence intensity for GluR1 is apparently higher for cells successfully transfected with sh-JNK1-1 compared with that of the neighboring GFP-negative cells (Fig. 6C, bottom left). Similar results were obtained with sh-JNK1-2 transfection (Fig. $6 D$ ). The quantified result is shown on the right $(p<0.001$ for both sh-JNK1 groups compared with the control group for JNK1 and for GluR1). These results together suggest that JNK1 negatively regulates GluR1 expression through the mediation of Hes-1 in cultured cortical neurons.

In separate sets of cortical cultures, we have further used the anti-HA antibody to stain JNK1 and have confirmed that the GFP-stained cell is actually the HA-stained cell (Fig. 6E). Similar experiment was performed with sh-JNK1 transfection using both GFP staining and anti-JNK1 antibody. Result revealed that both staining represents the same cell (Fig. 6F). These results verify that JNK1 indeed regulates GluR1 expression in the same neurons.

\section{JNK1 stabilizes the Hes-1 protein}

The above results demonstrated that JNK1 activation positively regulates Hes- 1 protein expression. But the mechanism underlying JNK1 regulation of Hes-1 is not known. This issue was examined here. We first conducted a time course experiment to study the effect of JNK1 activation by low dose of anisomycin (100 $\mathrm{ng} / \mathrm{ml}$ ) on Hes-1 mRNA expression. Results revealed that aniso- 
A
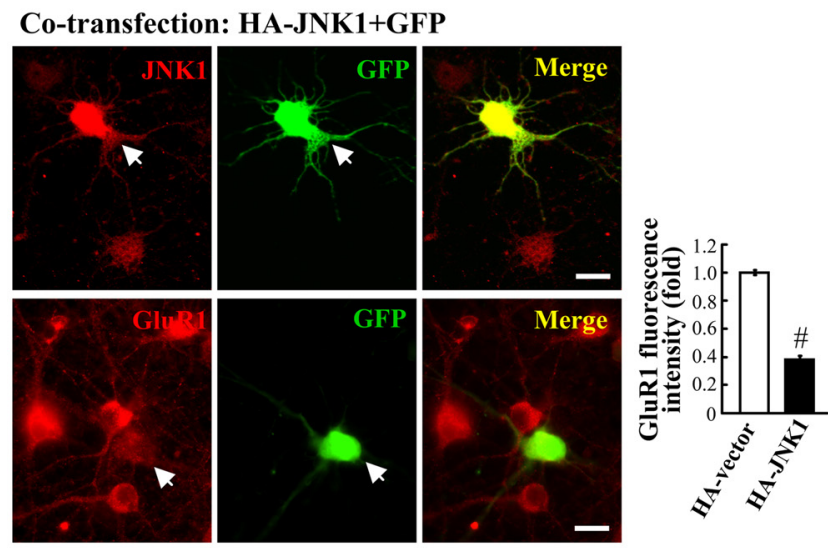

C

Co-transfection: sh-JNK1-1+GFP
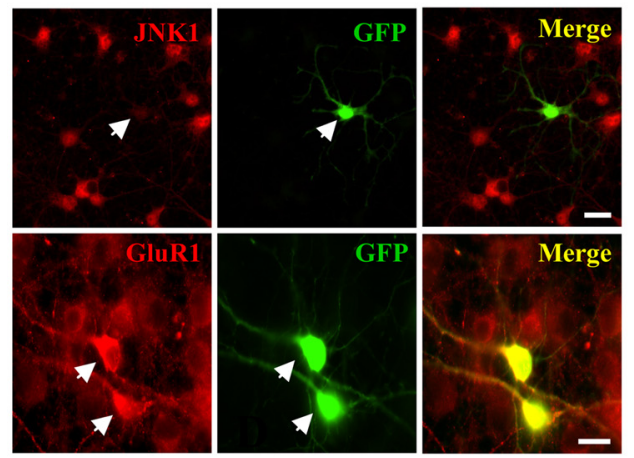

$\mathbf{E}$

Co-transfection: HA-JNK1+GFP
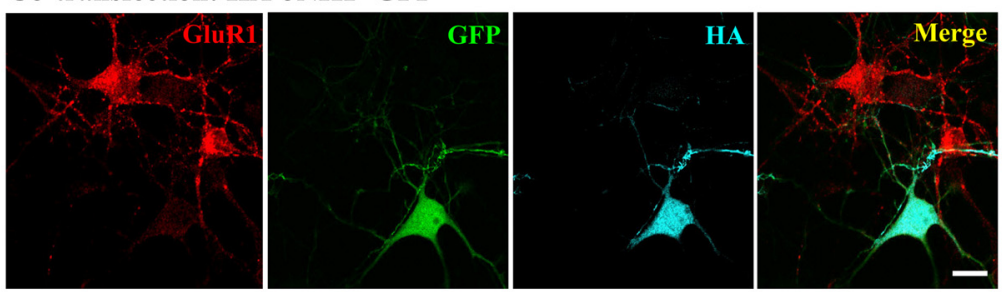

\section{Co-transfection: HA-JNK1+GFP+Control siRNA}
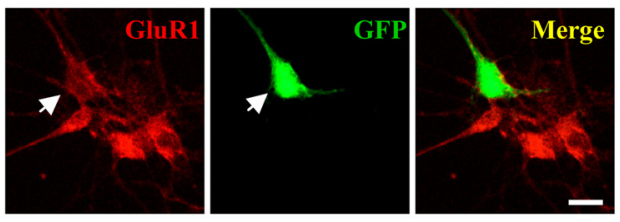

Co-transfection: HA-JNK1+GFP+Hes-1 siRNA
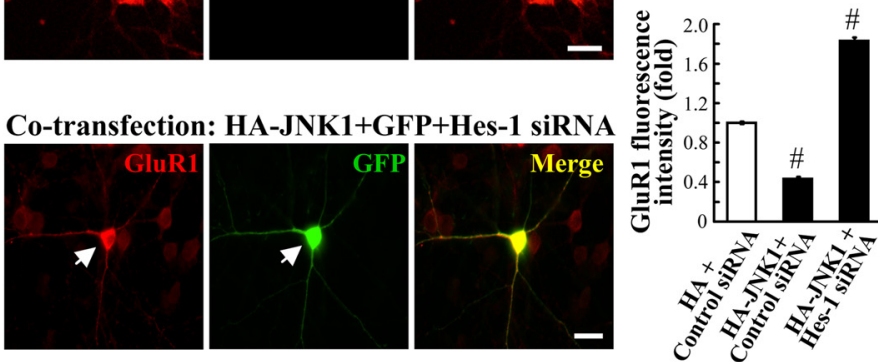

D
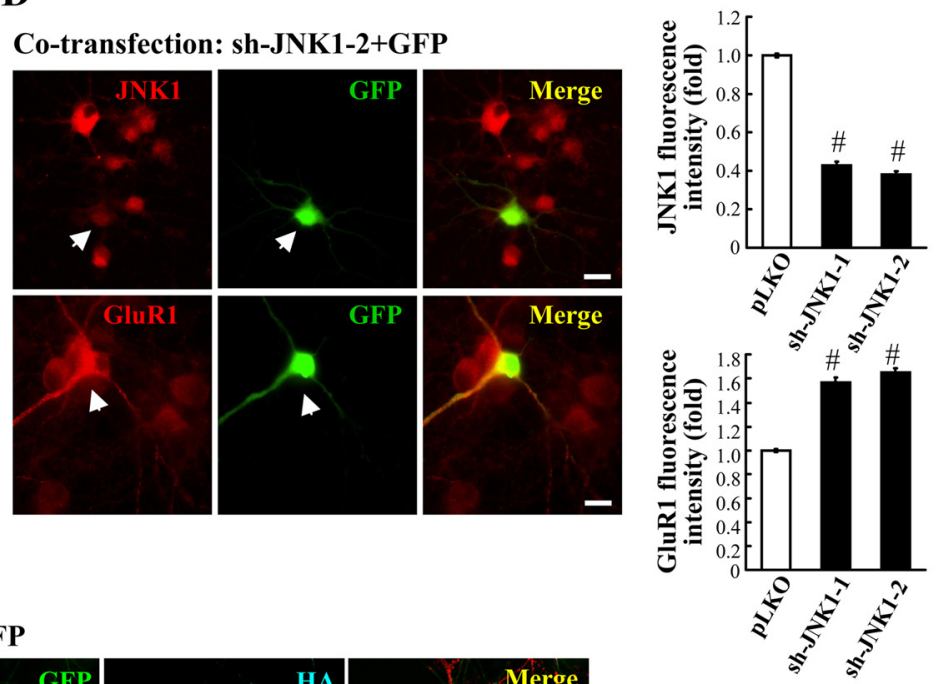

\section{F}

\section{Transfection: GFP-sh-JNK1-2}
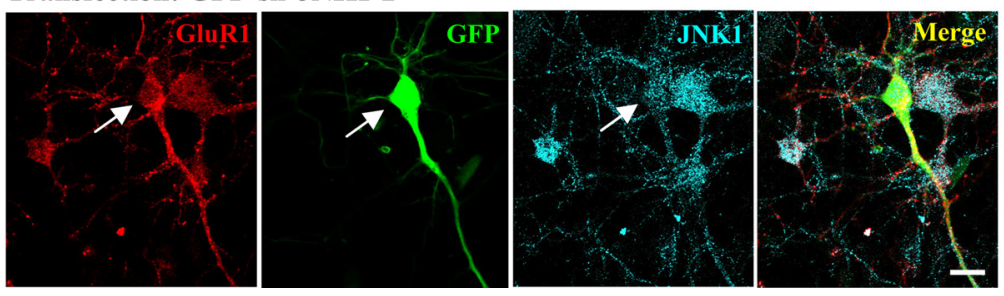

Figure 6. JNK1 decreases GluR1 expression level through the mediation of Hes-1 in cultured cortical neurons. A, HA-JNK1 plasmid and GFP plasmid were cotransfected to cultured cortical neurons, and immunofluorescence staining for JNK1 (red), GluR1 (red), and GFP (green) was examined. Scale bar, $10 \mu \mathrm{m}$. The quantified result is shown on the right. B, HA-JNK1 plasmid, GFP plasmid, and Hes-1 siRNA (or control siRNA) were cotransfected to cultured cortical neurons, and immunofluorescence staining for GluR1 (red) and GFP (green) was examined. Scale bars: top, $10 \mu \mathrm{m}$; bottom, 20 $\mu \mathrm{m}$. The quantified result is shown on the right. C, sh-JNK1 plasmid and GFP plasmid were cotransfected to cultured cortical neurons, and immunofluorescence staining for JNK1 (red), GluR1 (red), and GFP (green) was examined. $\boldsymbol{D}$, This experiment is the same as the above one except that the role of sh-JNK1-2 was examined. The quantified results are shown on the right. $\boldsymbol{E}$, HA-JNK1 plasmid and GFP plasmid were cotransfected to cultured cortical neurons and antibodies against GFP, HA, and GluR1 were used for immunofluorescence staining. These antibodies indeed stain the same neuron. $\boldsymbol{F}$, The GFP-tagged sh-JNK1-2 plasmid was transfected to cultured cortical neurons, and antibodies against GFP, JNK1, and GluR1 were used for immunofluorescence staining. These antibodies indeed stain the same neuron. GFP transfection and staining were performed for identification of the transfected neuron. Scale bar, $20 \mu \mathrm{m}$. The number of cells ranged from 15 to 20 for each group. Data are expressed as mean \pm SEM. ${ }^{\#} p<0.001$. 
A

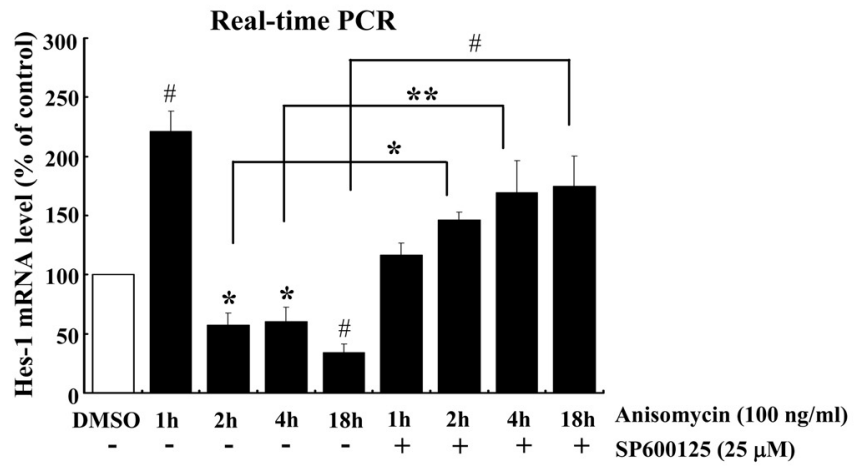

B

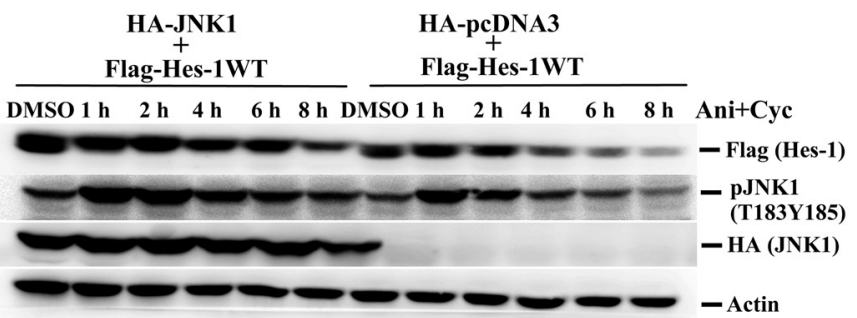

C
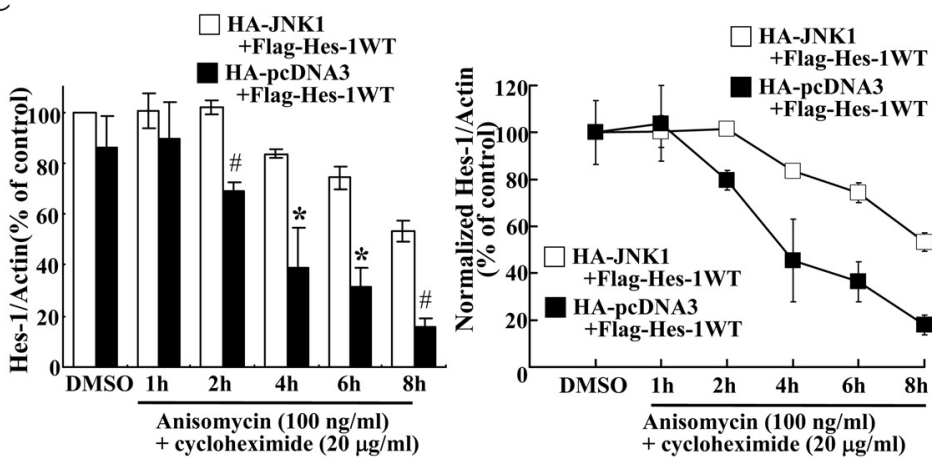

D

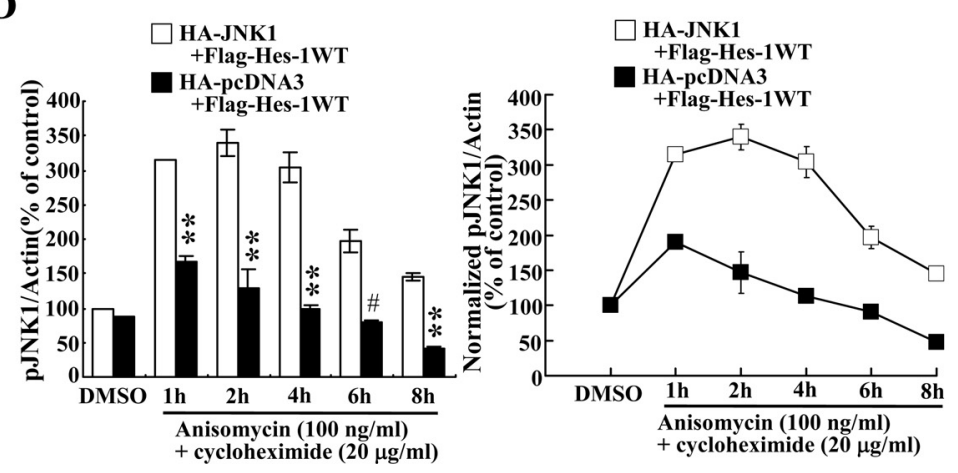

Figure 7. JNK1 stabilizes the Hes-1 protein. $A$, Anisomycin at $100 \mathrm{ng} / \mathrm{ml}$ (used as a JNK activator), alone or together with JNK inhibitor, was applied to cultured cortical neurons, and Hes- $7 \mathrm{mRNA}$ level was determined by real-time PCRat different time points. $\boldsymbol{B}$, Flag-Hes-1WT plasmid was cotransfected with the HA-JNK1WT plasmid (or control vector) to HEK293 cells with the addition of $100 \mathrm{ng} / \mathrm{ml}$ anisomycin to activate JNK1 and $20 \mu \mathrm{g} / \mathrm{ml}$ cycloheximide to inhibit translation. Their effects on the expression of Hes-1, JNK, and phospho-JNK were determined by Western blot. Ani, Anisomycin; Cyc, cycloheximide. C, The quantitative result for Hes-1 expression at each time point is shown on the left. The normalized degradation rate of the Hes-1 protein over time is shown on the right. $\boldsymbol{D}$, The quantitative result for $p$-JNK1 level at each time point is shown on the left. The normalized $p-J N K 1$ level over time is shown on the right. Experiments are in duplicate $\left(n=3\right.$ for each group). Data are expressed as mean \pm SEM. ${ }^{*} p<0.05,{ }^{* *} p<0.01$, and ${ }^{\#} p<0.001$. blocked the effect of anisomycin but also increased Hes-1 mRNA level in a timedependent manner (Fig. 7A) $(p<0.05$, $p<0.01$, or $p<0.001$ compared with the corresponding anisomycin group). These results suggest that JNK1 activation inhibits the transcription of the Hes-1 gene.

The opposite effect of JNK1 activation on Hes-1 mRNA and protein expression further suggests that JNK1 may stabilize the Hes-1 protein. To test this hypothesis, we have cotransfected the Flag-Hes-1WT plasmid and the HA-JNK1WT plasmid (or the control plasmid) to HEK293 cells with the addition of the translation inhibitor cycloheximide at $20 \mu \mathrm{g} / \mathrm{ml}$. Low concentration of anisomycin $(100 \mathrm{ng} / \mathrm{ml})$ was also added to activate JNK1. The expression of Hes-1, JNK1, and phospho (p)-JNK1 was examined. Results revealed that Hes-1 protein level gradually decreased over time after blockade of protein translation, but its expression level was higher and stayed longer after HA-JNK1WT cotransfection (Fig. 7B). The quantitative result for Hes- 1 expression at each time point is shown in Figure $7 C$, left $(p<0.05$ or $p<0.001$ for various time points). The degradation rate of the Hes-1 protein level over time is shown in Figure $7 C$, right. To compare the degradation rate of Hes-1 over time between these two groups, data were normalized to $100 \%$ at time 0 for both groups. These results revealed that the Hes-1 protein level gradually decreased over time in control group, but it decreased more slowly in HA-JNK1cotransfected group (Fig. 7C). Meanwhile, the phosphorylation level of JNK1 was increased and lasted up to $8 \mathrm{~h}$ after HAJNK1WT transfection (Fig. 7B). The quantitative result for $\mathrm{p}$-JNK1 level at each time point is shown in Figure $7 D$, left ( $p<0.01$ or $p<0.001$ for various time points). Similarly, for the purpose of comparison of p-JNK1 level over time between these two groups, data were normalized to $100 \%$ at time 0 for both groups. The normalized result showed that the p-JNK1 level gradually decreased overtime in Flag-Hes-1WT group, but it decreased more slowly in HA-JNK1cotransfected group (Fig. 7D, right). Transfection of HA-JNK1WT was confirmed by Western blot against the HA tag (Fig. 7B). These results together suggest that JNK1 increased the expression of Hes-1 possibly due to JNK1 stabilization of Hes-1. mycin enhanced Hes-1 mRNA expression $1 \mathrm{~h}$ later $(p<0.001)$. But unlike the effect of anisomycin on Hes-1 protein expression (Fig. 4D), anisomycin dramatically decreased Hes-1 mRNA level since $2 \mathrm{~h}$ later and it lasted up to $18 \mathrm{~h}(p<0.05$ or $p<0.001)$. Coadministration of the JNK1 inhibitor SP600125 not only
JNK1 interacts with Hes-1 and phosphorylates Hes-1 at Ser-263 in vitro and in vivo

The above results demonstrated that JNK1 stabilizes the Hes-1 protein, but how JNK1 stabilizes Hes-1 is not known and was examined here. We first studied whether JNK1 is associated with 
Hes-1. Flag-JNK1 and His-Hes-1 plasmids were cotransfected to HEK293 cells. Co-IPs of Flag and Western blot were performed. Results revealed that JNK1 interacts with Hes-1 and vice versa (Fig. 8A). Next, we examined whether Hes-1 is a substrate for JNK1. The result of in vitro kinase for several candidate phosphorylation sites on Hes-1 for JNK1 was shown in Figure $8 B$. The result suggests that JNK1 may directly phosphorylate Hes-1 at Ser-263. To confirm this result, we have synthesized the phospho-263 Hes-1 peptide as the antigen and raised antibody against this residue to perform additional in vitro kinase assay and Western blot experiments. The result revealed that JNK1 consistently phosphorylates Hes-1 at Ser-263, and this phosphorylation was confirmed by Western blot when the pSer-263 antibody was used (Fig. 8C).

Next, we examined whether JNK1 phosphorylates Hes-1 in vivo. HA-JNK1 and Flag-Hes-1 plasmids were cotransfected to HEK293 cells. Anisomycin $(20 \mu \mathrm{g} / \mathrm{ml})$ was added to activate JNK signaling and also inhibit endogenous translation of the Hes- 1 protein (Frey et al., 1988). The result revealed that JNK1 phosphorylates Hes-1 at Ser-263 in HEK293 cells, and this effect lasted up to $8 \mathrm{~h}$ (Fig. 8D). The quantitative result of pSer-263 Hes-1 level at each time point is shown on the left (Fig. $8 D$, bottom panel) $(p<0.05, p<0.01$, or $p<$ 0.001 for different time points). The normalized pS263 Hes-1 level over time is shown on the right (Fig. $8 D$, bottom panel). These results indicated that transfection of HA-JNK1 slowed down the degradation rate of the Hes-1 protein.

Last, we examined whether JNK1 enhances the phosphorylation of Hes-1 in cortical neurons and whether this affects the expression of Hes-1 and GluR1. A low dose of anisomycin (100 $\mathrm{ng} / \mathrm{ml}$ ) was used to activate JNK1, and the expression of pS263Hes-1, p-JNK1, Hes-1, and GluR1 was examined by Western blot. Quantitative results revealed that JNK1 activation increased the phosphorylation level of Ser-263 Hes- 1 and JNK1 over a wide time range examined ( $p<0.05, p<0.01$, or $p<0.001$ for different time points). JNK1 activation also increased the expression of Hes- 1 and stabilized Hes- 1 over time, but it decreased the expression of GluR1 in cortical neurons ( $p<0.01$ for 4 and $18 \mathrm{~h}$ ) (Fig. $8 E$ ). These results together suggest that JNK1 phosphorylates Hes-1 at Ser-263 and stabilizes Hes-1, and this is associated with decreased GluR1 expression.

\section{JNK1 stabilizes Hes-1 through phosphorylation of Hes-1 at Ser-263}

The above results suggest that JNK1 phosphorylates Hes-1 and stabilizes Hes-1, but it is not known whether JNK1 phosphorylation of Hes-1 mediates this effect. This issue was examined here. Flag-Hes-1WT plasmid or Flag-Hes-1S263A mutant plasmid $(0.2 \mu \mathrm{g}$ each) was transfected to HEK293 cells. Anisomycin (100 $\mathrm{ng} / \mathrm{ml}$ ) was added to the cell to activate JNK1, and cycloheximide $(20 \mu \mathrm{g} / \mathrm{ml})$ was added to inhibit translation. The expression of Hes- 1 and its degradation rate over time (Hes-1 protein level over that of the corresponding control group at each time point) was examined by Western blot. The quantitative results for Hes-1 expression at each time point is shown in Figure 9A, bottom left. The normalized degradation rate of the Hes-1 protein over time is shown in Figure 9A, bottom right. These results showed that Hes-1 protein level gradually decreased over time, but Hes1S263A protein level decreased more rapidly than the Hes-1WT protein (Fig. $9 A$, bottom panels) $(p<0.05$ or $p<0.01$ for various time points). The effectiveness of Hes-1WT transfection was confirmed by an apparent increase in Hes-1 protein level comparing the two control (DMSO) groups. The effectiveness of Hes1S263A transfection was confirmed by an apparent loss of pS263-
Hes-1 protein expression (Fig. 9A, top panel). This result suggests that Hes-1 phosphorylation at Ser-263 stabilizes Hes-1.

Next, we examined whether inactivation of JNK1 reduced the phosphorylation of Hes-1 at Ser-263 and decreased the stability of Hes-1. The Flag-Hes-1WT plasmid was transfected to HEK293 cells $(0.2 \mu \mathrm{g})$, and SP600125 $(25 \mu \mathrm{M})$ was added to the cell $24 \mathrm{~h}$ later. Anisomycin $(100 \mathrm{ng} / \mathrm{ml})$ was added to activate JNK1, and cycloheximide $(20 \mu \mathrm{g} / \mathrm{ml})$ was added to inhibit translation. The control group received DMSO treatment. Results revealed that SP600125 apparently decreased the phosphorylation level of Hes-1 at Ser-263 (Fig. 9B, top panel). The quantitative result for Hes- 1 expression at each time point is shown in Figure 9B, bottom left. The normalized degradation rate of the Hes- 1 protein over time is shown in Figure $9 B$, bottom right. These results revealed that SP600125 decreased Hes-1 expression and accelerated the degradation rate of the Hes- 1 protein (Fig. $9 B$, bottom panels) ( $p<0.05, p<0.01$, or $p<0.001$ for various time points). However, the degradation rate for Hes-1 becomes similar when SP600125 (25 $\mu \mathrm{M})$ was added to either Flag-Hes-1WTtransfected or Flag-Hes-1S263A-transfected HEK293 cells because JNK1 activity was inhibited by SP600125 (Fig. 9C) ( $p>$ 0.05 except at $2 \mathrm{~h}$ ). These results together suggest that JNK1 phosphorylation of Hes- 1 at Ser-263 plays an important role in stabilization of the Hes-1 protein.

\section{JNK decreases GluR1 expression at the synapse through the mediation of Hes-1}

The above results demonstrated that JNK1 negatively regulates GluR1 expression in cultured cortical neurons through the mediation of Hes-1. But all these results were obtained on the soma, whereas GluR1 plays an important role in synaptic plasticity; one would expect that the same results should occur at the synapse. In this experiment, we examined whether the same regulation mechanisms for GluR1 expression also take place at synapses. Anisomycin and SP600125 were added to cultured cortical neurons to activate and to inhibit JNK activity, respectively. Eighteen hours later, cultured cortical neurons were separated to different fractions, including total lysate, S2 fraction, and P2 fraction according to the method of Gurd et al. (1974) with some modifications. A representative gel pattern is shown in Figure $10 \mathrm{~A}$, left. Quantitative analysis revealed that anisomycin decreased GluR1 expression $(p<0.01)$, whereas SP600125 increased GluR1 expression $(p<0.05)$ in $\mathrm{P} 2$ fraction. But the suppressing effect of anisomycin on GluR1 expression is blocked by SP600125 cotreatment ( $p<0.001$ compared with the anisomycin group) (Fig. $10 \mathrm{~A}$, right). Western blotting of SNAP-25 (a presynaptic marker) and PSD-95 (a postsynaptic marker) was performed to verify the P2 fraction preparation. GluR1, SNAP-25, and PSD-95 were not present in the $\mathrm{S} 2$ fraction.

In the next experiment, we examined the effect of Hes- 1 plasmid transfection and Hes-1 siRNA transfection on GluR1 expression at synapse. Because the transfection efficiency is low in cultured neurons, in this experiment and the following experiment, plasmid DNA and Hes-1 siRNA were transfected to rat hippocampal CA1 area in vivo. Rats were cannulated in CA1 area bilaterally. Plasmid DNA was diluted in 5\% glucose to a stock concentration of 2.77 $\mu \mathrm{g} / \mu \mathrm{l}$. Branched polyethyleneimine (PEI) of $25 \mathrm{kDa}$ (SigmaAldrich), used as the nonviral transfection reagent (Tai et al., 2011), was diluted to $0.1 \mathrm{~m}$ concentration in $5 \%$ glucose and added to the DNA solution [PEI $(0.45 \mu \mathrm{l}) / \mathrm{DNA}(0.55 \mu \mathrm{l})]$. Immediately before injection, $0.1 \mathrm{M}$ PEI was added to reach a ratio of PEI nitrogen per DNA phosphate equals to 10 . For Hes-1 siRNA transfection, Hes- 1 siRNA was diluted in distilled water to a stock concentration of 20 
A

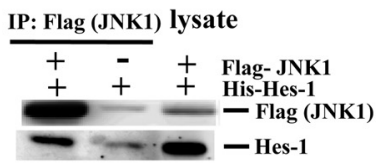

IP: Flag (Hes-1) lysate

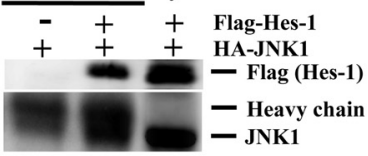

B

\section{In vitro kinase assay}

Active JNK1

His-Hes-1 WT WT 12A 17A 24A 30A 172A 201A 263A

$\gamma$-32P-ATP

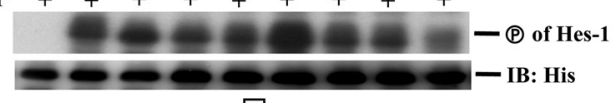

S12: SSSRP

T17: AATPA

T24: NTPPD

T30: PKTAS

S172: PPSGP

S201: PQSAP

S263: Aysps

C

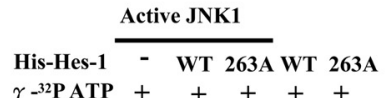

Antigen peptide

SV-GPNAVSPSSG-SS-rat SV-GPNAVSPSSG-SS-mouse

SV-GPNAUSPSSG-PS-human S263

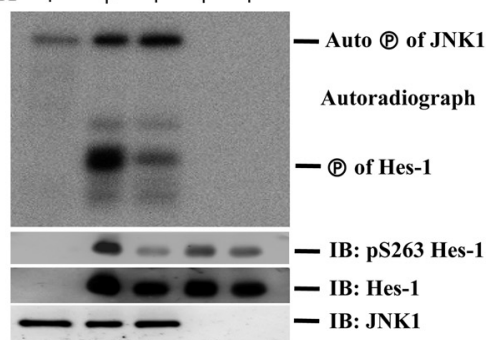

D
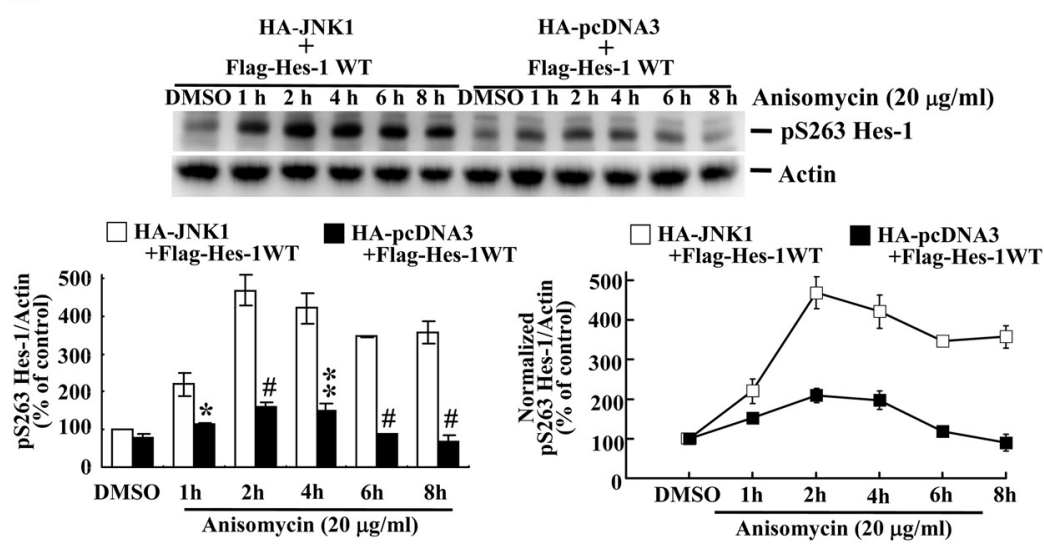

$\mathbf{E}$
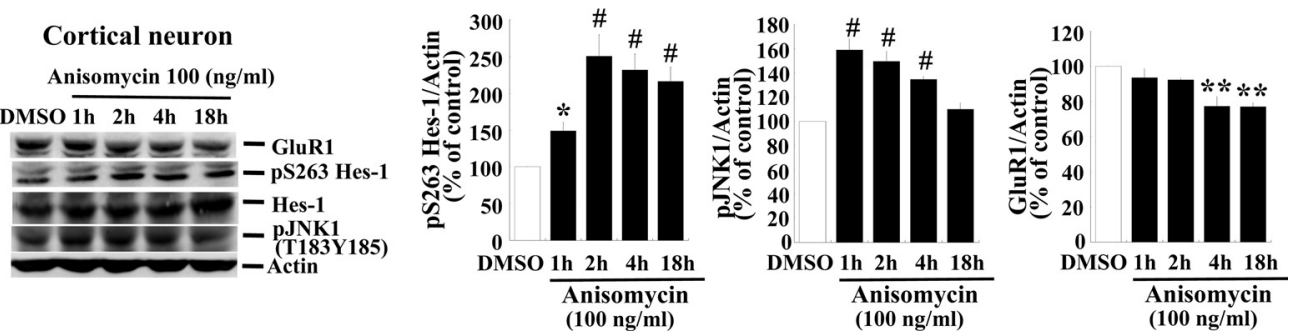

Figure 8. JNK1 interacts with Hes-1 and phosphorylates Hes-1 at Ser-263. A, Co-IP and immunoblot showing the association of JNK1 with Hes-1 in HEK293 cells. B, Several candidate phosphorylation sites on Hes-1 were identified based on the consensus motif of JNK1 substrate (proline-dependent serine and threonine) that includes Ser-12, Thr-17, Thr-24, Thr-30, Ser-172, Ser-201, and Ser-263. The Hes-1 wild-type protein and mutant proteins (mutated at the above residues individually) purified from E. coli. were subjected to in vitro kinase assay for JNK1. C, In vitro kinase assay for JNK1 phosphorylation of Hes-1 at Ser-263 was performed and confirmed by Western blot using phospho-Ser-263-specific antibody. Sequences of antigen peptide from rat, mouse, and human containing Ser-263 (which is conserved) are shown (left). D, Flag-Hes-1WT plasmid and HA-JNK1 plasmid (or control plasmid) were cotransfected to HEK293 cells with $20 \mu \mathrm{g} / \mathrm{ml}$ anisomycin added to both activate JNK1 and inhibit translation. Quantitative result for pS263 Hes-1 expression at each time point is shown on the bottom left panel. The normalized pS263 Hes-1 level over time is shown on the bottom right panel. $\boldsymbol{E}$, Anisomycin at $100 \mathrm{ng} / \mathrm{ml}$ (used to activate JNK) was applied to cultured cortical neurons, and its effect on the expression of GluR1, Hes-1, pS263 Hes-1, and p-JNK1 at different time points is shown. The quantitative results are shown on the right.P (in circle), Phosphorylation; IB, immunoblot. Experiments are in duplicate ( $n=3$ for each group). Data are expressed as mean \pm SEM. ${ }^{*} p<0.05,{ }^{* *} p<0.01$, and ${ }^{\#} p<0.001$.

$\mu \mathrm{M}$. PEI was diluted to $0.1 \mathrm{M}$ concentration in $5 \%$ glucose and mixed with Hes-1 siRNA [PEI $(0.5 \mu \mathrm{l}) /$ Hes-1 siRNA $(0.5 \mu \mathrm{l})]$. The CA1 tissue was punched out $48 \mathrm{~h}$ after transfection and the P2 fraction was prepared in the same way. A representative gel pattern is shown in Figure $10 B$, left. Quantitative analysis revealed that Hes-1WT plasmid transfection decreased GluR1 expression $(p<0.01)$, but Hes-1 siRNA transfection increased GluR1 expression $(p<0.05)$ in P2 fraction of the CA1 tissue (Fig. 10B, right). 
A

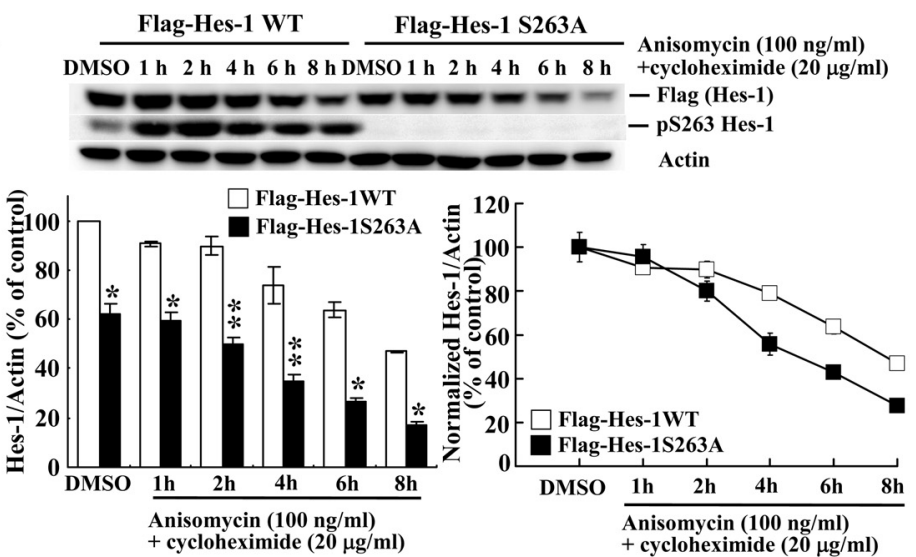

B
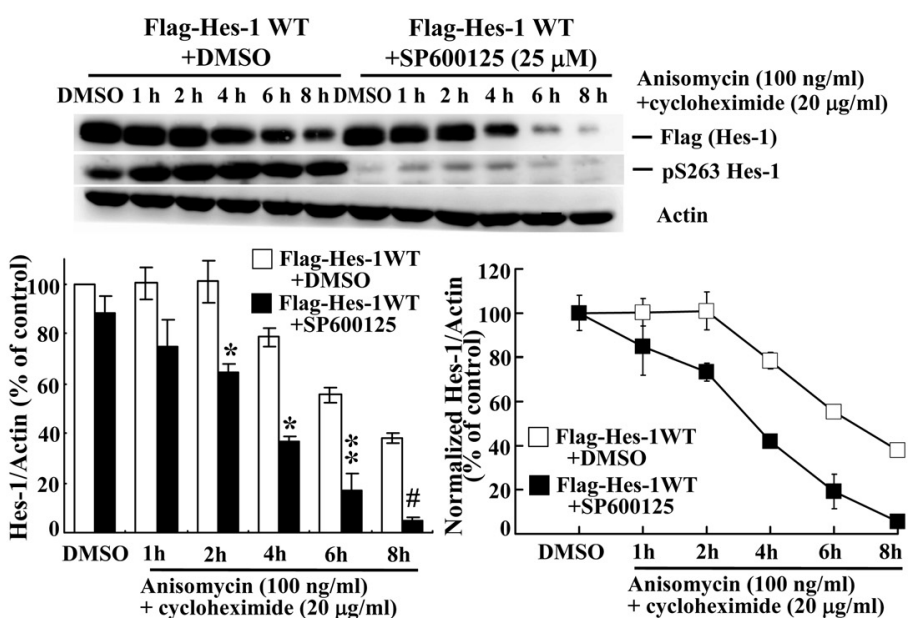

C

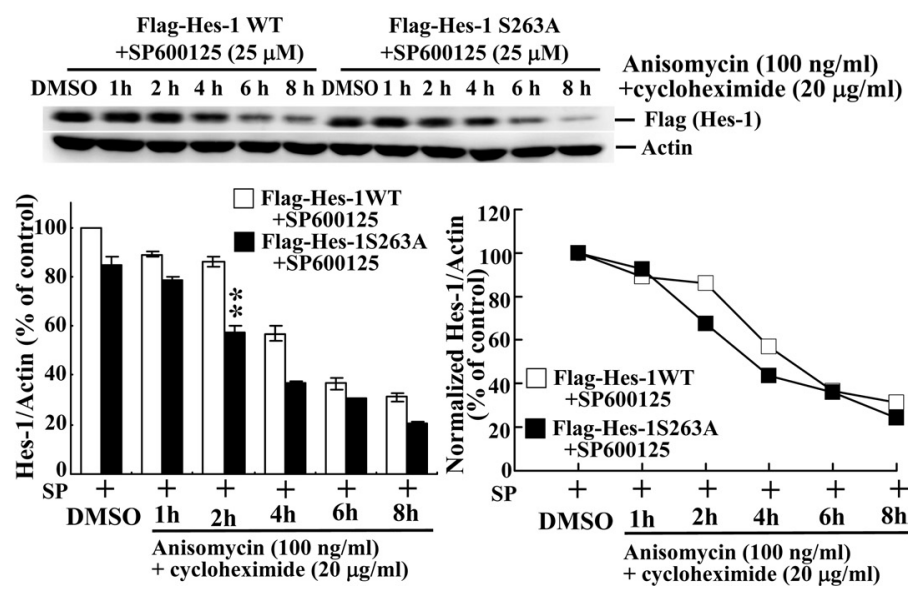

Figure 9. JNK1 stabilizes Hes-1 through phosphorylation of Hes-1 at Ser-263. A, Flag-Hes-1WT or Flag-Hes-1S263A mutant plasmid was transfected to HEK293 cells with the addition of $100 \mathrm{ng} / \mathrm{ml}$ anisomycin to activate JNK1 and $20 \mu \mathrm{g} / \mathrm{ml}$ cycloheximide to inhibit translation. The expression level of Hes- 1 and pS263Hes- 1 was determined by Western blot (top panel). The quantitative result for Hes-1 expression at each time point is shown on the left. The normalized degradation rate of the Hes- 1 protein over time is shown on the right. $\boldsymbol{B}$, Flag-Hes-1WT plasmid was transfected to HEK293 cells with the addition of $100 \mathrm{ng} / \mathrm{ml}$ anisomycin and 20 $\mu \mathrm{g} / \mathrm{ml}$ cycloheximide, together with JNK inhibitor or with DMSO as a control. The expression level of Hes-1 and pS263Hes-1 was determined by Western blot (top panel). The quantitative result for Hes-1 expression at each time point is shown on the left. The normalized degradation rate of the Hes-1 protein over time is shown on the right. C, Flag-Hes-1WT or it mutant plasmid Hes1 S263A was transfected to HEK 293 cells with the addition of $100 \mathrm{ng} / \mathrm{ml}$ anisomycin and $20 \mu \mathrm{g} / \mathrm{ml}$ cycloheximide together with the JNK inhibitor. The expression level of Hes- 1 was examined by Western blot (top panel). The quantitative result for Hes-1 expression at each time point is shown on the left. The normalized degradation rate of the Hes- 1 protein over time is shown on the right. SP, SP600125 $(25 \mu \mathrm{M})$. Experiments are in duplicate ( $n=3$ for each group). Data are expressed as mean \pm SEM. ${ }^{*} p<0.05,{ }^{* *} p<$ 0.01 , and ${ }^{\#} p<0.001$.
Next, we examined whether the suppressing effect of JNK1 on GluR1 expression at synapses is mediated through JNK1 phosphorylation of Hes-1 at Ser-263. The JNK1 plasmid was transfected to the CAl area, alone or in combination with the Hes-1 plasmid or Hes-1S263A plasmid. Western blot was performed $48 \mathrm{~h}$ after transfection. A representative gel pattern is shown in Figure $10 C$, left. Quantitative analysis revealed that JNK1 consistently suppressed GluR1 expression in $\mathrm{P} 2$ fraction $(p<0.001)$. This effect was further potentiated by Hes-1WT plasmid cotransfection $(p<0.001$ compared with the JNK1 group), but Hes1 S263A cotransfection reversed this effect $(p<0.01$ compared with JNK1 plus Hes-1 group) (Fig. 10C, right). SNAP-25 and PSD-95 were used as presynaptic and postsynaptic markers, respectively. Coimmunoprecipitation and Western blot experiments were performed to confirm the transfection and expression of JNK1 and Hes-1 (or Hes$1 \mathrm{~S} 263 \mathrm{~A})$ in CA1 area for the above experiments (Fig. 10D).

The above results showed that JNK1 regulates GluR1 expression at synapse. In this experiment, we further examined whether JNK1 regulates GluR1 expression on postsynaptic membrane and whether this is also mediated through Hes-1. Various plasmids and Hes-1 siRNA were transfected to cultured cortical neurons, and the expression of GluR1 on dendrites was examined by immunofluorescence staining. Immunofluorescence staining of PSD-95 was used as the marker for postsynaptic dendrites. Only GluR1 expression that is colocalized with PSD-95 expression was taken for quantification. Results from Figure 11, $A$ and $B$, revealed that transfection of Flag-GluR1 plasmid markedly increased GluR1 fluorescence intensity on dendrites $(p<0.001)$, whereas transfection of sh-GluR1 markedly decreased GluR1 fluorescence intensity on dendrites $(p<0.001)$, respectively. Images at a higher magnification from the selected rectangular areas are shown at the bottom panels.

Next, we examined the effect of Hes-1 on GluR1 expression. Results revealed that transfection of Flag-Hes-1 plasmid decreased GluR1 fluorescence intensity on dendrites in cultured cortical neurons $(p<0.001)$. Images at a higher magnification are shown at the bottom panels (Fig. 11C). Next, we examined the effect of JNK1 on GluR1 expression on dendrites. HA-JNK1 plasmid or sh-JNK1 was transfected to cultured cortical neurons. Results from Figure $11 D$ revealed that transfection of HA-JNK1 decreased GluR1 fluorescence intensity on dendrites $(p<0.001)$, but 
A
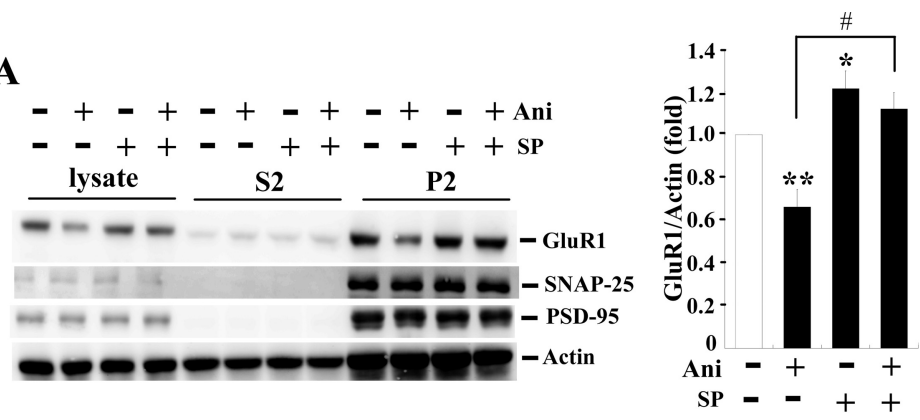

B
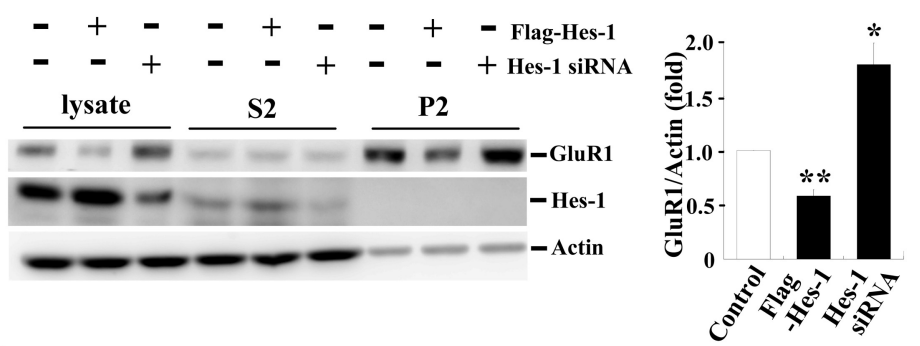

C

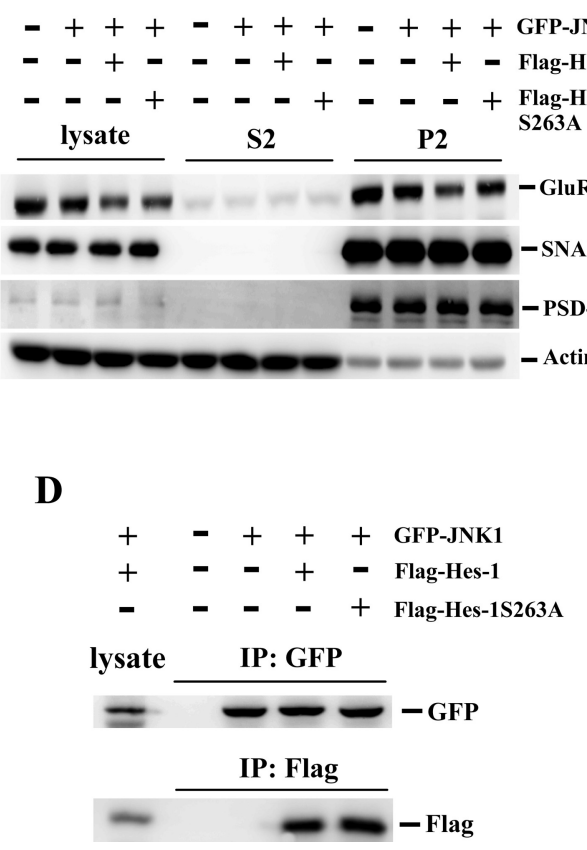

Figure 10. JNK decreases GluR1 expression at the synapse through the mediation of Hes-1.A, Anisomycin at $100 \mathrm{ng} / \mathrm{ml}$ (used as a JNK activator), alone or together with SP600125 (25 $\mu \mathrm{M})$, a JNK inhibitor, was applied to cultured cortical neurons. Total lysate, S2 fraction, and P2 fraction were prepared, and GluR1 expression was determined by Western blot. SNAP-25 and PSD-95 were used as presynaptic and postsynaptic markers, respectively. Ani, Anisomycin; SP, SP600125. B, Flag-Hes-1WT plasmid or Hes-1 siRNA was transfected to rat hippocampal CA1 area directly. Total lysate, $S 2$ and P2 fractions from CA1 tissues were prepared $48 \mathrm{~h}$ after transfection for Western blot analysis of Hes-1 and GluR1 expression. C, GFP-JNK1 plasmid was cotransfected with Flag-Hes-1WT plasmid or Flag-Hes-15263A mutant plasmid to rat CA1 area. Total lysate, S2 and P2 fractions from CA1 tissues were prepared 48 h after transfection for Western blot determination of GluR1 expression. SNAP-25 and PSD-95 were used as presynaptic and postsynaptic markers, respectively. $\boldsymbol{D}$, Coimmunoprecipitation and immunoblotting experiment was performed to confirm the transfection and expression of plasmid DNA in rat CA1 area. Data are expressed as mean \pm SEM. Experiments are in triplicate $(n=3$ for each group). ${ }^{*} p<0.05,{ }^{* *} p<0.01$, and ${ }^{\#} p<0.001$.

transfection of sh-JNK1 increased GluR1 fluorescence intensity on dendrites $(p<0.001)$ (Fig. 11E). Images at a higher magnification are shown at the bottom panels.

Last, we examined whether JNK1 suppressed the expression of GluR1 on dendrites through the mediation of Hes-1. HA-JNK1 was transfected alone, or together with Hes-1 siRNA to cultured cortical neurons. Results revealed that transfection of HA-JNK1 consistently decreased GluR1 fluorescence intensity on dendrites $(p<0.001)$, but this suppressing effect of HA-JNK1 was blocked by cotransfection with Hes-1 siRNA ( $p<0.001$ comparing the HA-JNK1 plus Hes-1 siRNA group with HA-JNK1 group) (Fig. $11 \mathrm{~F}$ ). Images at a higher magnification are shown at the bottom panels. Only images for the control group and the HA-JNK1 plus Hes-1 siRNA group are shown.

JNK1 decreases AMPA-evoked calcium influx through the mediation of Hes-1 and through Hes-1 phosphorylation at Ser-263

The above results demonstrated that JNK1 suppressed GluR1 promoter activity through JNK1 phosphorylation of Hes- 1 at Ser-263 and, consequently, the stabilization of Hes-1. In this series of experiments, we examined the physiological significance of this signaling pathway involved in calcium influx in cultured cortical neurons. The Fluo-4 AM dye was used as a marker for visualization of the calcium image, and the Fluo-4 AM fluorescence intensity is proportional to the concentration of calcium in the cell. For each culture well, $50 \mu \mathrm{M}$ APV was added to block the NDMA receptor and $100 \mu \mathrm{M}$ nifedipine was added to block calcium channel so that the effect of exogenously applied AMPA could be observed. In the first experiment, AMPA $(30 \mu \mathrm{M})$ was added to the culture medium to induce calcium influx, and the fluorescence intensity for calcium was measured before and at various time points after AMPA treatment. Results revealed that application of AMPA induced a rapid and apparent increase $(\sim 2.25$-fold $)$ in calcium influx. The maximum effect was observed at $30 \mathrm{~s}$, and it gradually declined thereafter (Fig. 12A). The representative fluorescence images are shown in the top panel of Figure $12 \mathrm{~B}$. Results revealed that the fluorescence intensity for calcium is apparently higher at $30 \mathrm{~s}$ after AMPA treatment compared with that at the basal level. But this effect of AMPA was blocked after coadministration of the AMPA receptor antagonist NBQX $(20 \mu \mathrm{M})$ (Fig. 12B, bottom panel).

Next, we examined the roles of GluR1 and Hes-1 involved in calcium influx. Various HA- or Flag-tagged plasmids $(0.6 \mu \mathrm{g})$ and/or siRNAs (40 $\mathrm{nM})$ together with the pCMV-DsRED plasmid $(0.6 \mu \mathrm{g})$ were cotransfected to cultured cortical neurons and their effects on fluorescence intensity of calcium were measured. Control groups received vector plasmid transfection and/or control siRNA transfection. Cells that showed orange color in the merged images are cells successfully transfected with the plasmid and/or siRNA. 
A Co-transfection: GFP+Flag-GluR1
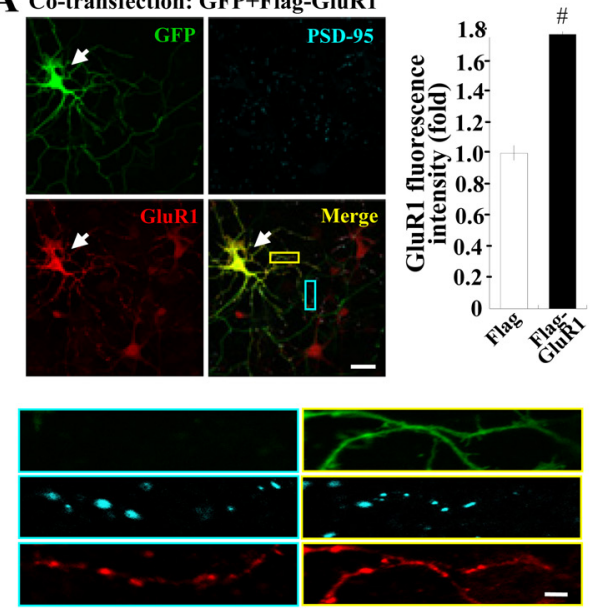

C Co-transfection: GFP+Flag-Hes-1
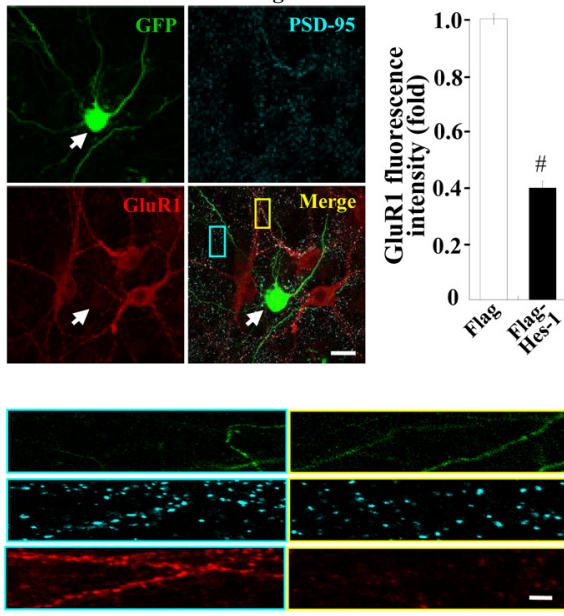

$E_{\text {Co-transfection: GFP+sh-JNK1 }}$
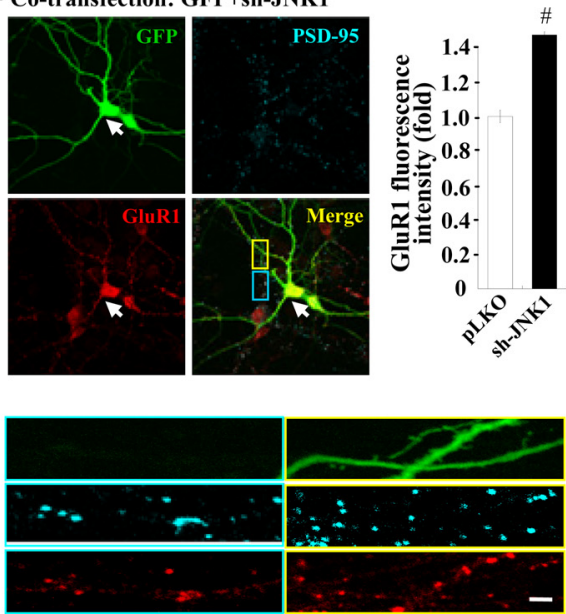

B Co-transfection: GFP+sh-GluR1
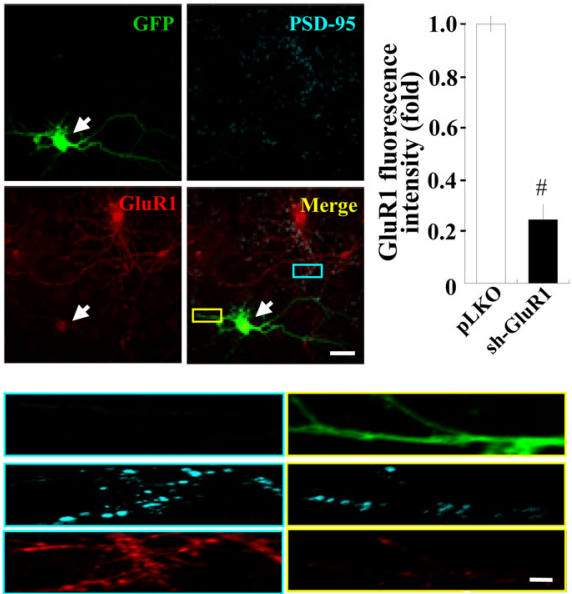

D Co-transfection: GFP+HA-JNK1
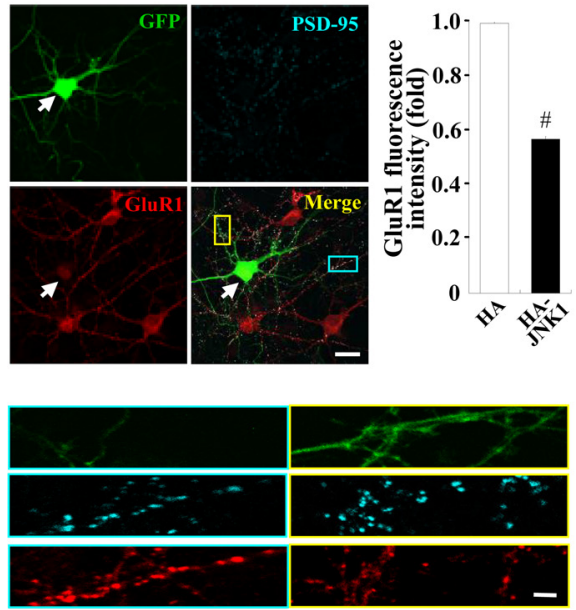

$\mathbf{F}$
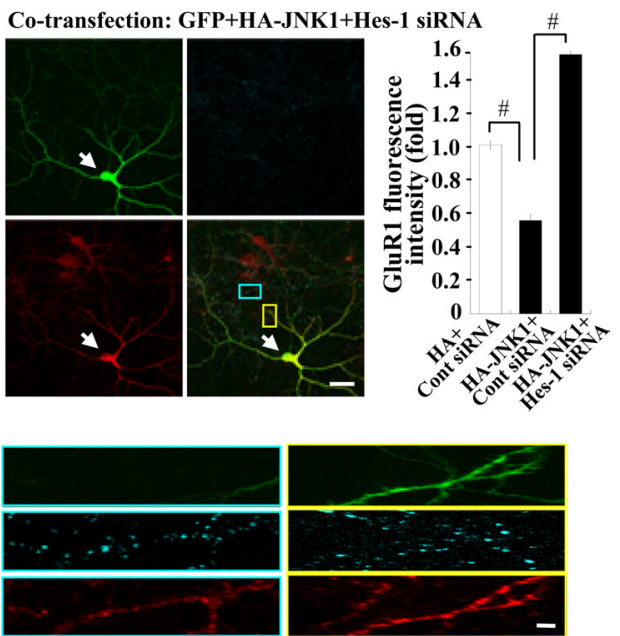

Figure 11. JNK1 decreases GluR1 expression at synaptic membrane through Hes-1. A, Flag-GluR1 plasmid and GFP plasmid were cotransfected to cultured cortical neurons and immunofluorescence staining for GluR1 (red), PSD-95 (blue), and GFP (green) was examined. The quantified result is shown on the right. $\boldsymbol{B}$, sh-GluR1 plasmid and GFP plasmid were cotransfected to cultured cortical neurons, and immunofluorescence staining for GluR1 (red), PSD-95 (blue), and GFP (green) was examined. The quantified result is shown on the right. C, Flag-Hes-1 plasmid and GFP plasmid were cotransfected to cultured cortical neurons and immunofluorescence staining for GluR1 (red), PSD-95 (blue), and GFP (green) was examined. The quantified result is shown on the right. D, HA-JNK1 plasmid and GFP plasmid were cotransfected to cultured cortical neurons, and immunofluorescence staining for GluR1 (red), PSD-95 (blue), and GFP (green) was examined. The quantified result is shown on the right. $\boldsymbol{E}$, sh-JNK1 plasmid and GFP plasmid were cotransfected to cultured cortical neurons, and immunofluorescence staining for GluR1 (red), PSD-95 (blue), and GFP (green) was examined. The quantified result is shown on the right. $\boldsymbol{F}$, HA-JNK1 plasmid, GFP plasmid, and Hes-1 siRNA were cotransfected to cultured cortical neurons, and immunofluorescence staining for GluR1 (red), PSD-95 (blue), and GFP (green) was examined. The quantified result is shown on the right. The yellow rectangular area selected from a transfected neuron and the blue rectangular area selected from a neighboring nontransfected neuron were enlarged at the bottom panels for better visualization of GluR1 (red) and PSD-95 (blue) on dendrites at a higher magnification. GFP transfection and staining was performed for identification of the transfected neuron. Scale bars: top panels, $20 \mu \mathrm{m}$; bottom panels, $0.2 \mu \mathrm{m}$. Data are expressed as mean \pm SEM. Experiments are in triplicate. The number of cells ranged from 15 to 20 for each group. ${ }^{p} p<0.001$. 


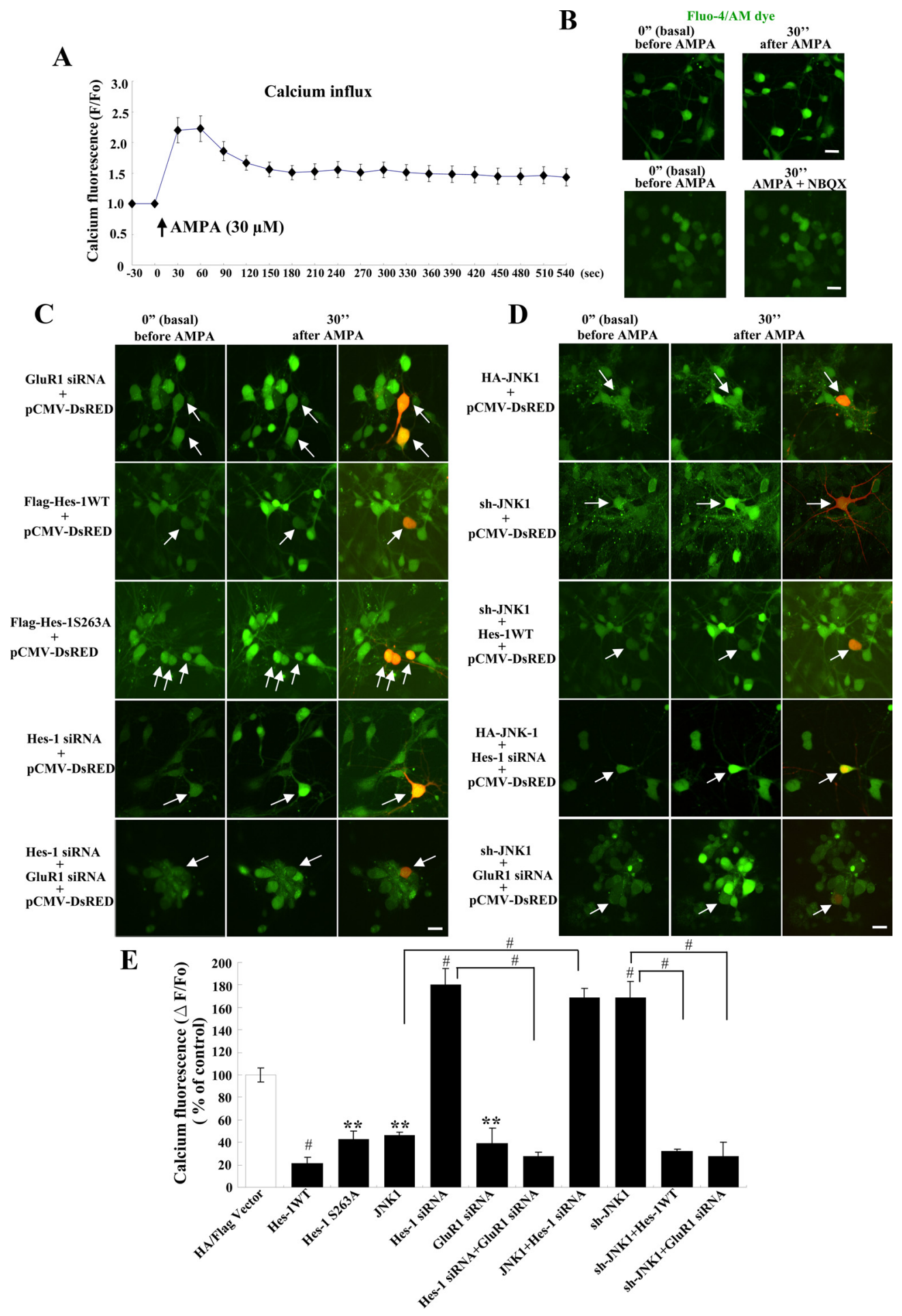

Figure 12. JNK1 decreases AMPA-evoked calcium influx through the mediation of Hes-1 and through Hes-1 phosphorylation at Ser-263. A, Using the Fluo-4 dye as a marker for intracellular calcium, the time course effect of AMPA-evoked calcium influx was measured and quantified in cultured cortical neurons. $F / F_{0}$ represents the ratio of fluorescence signal at each time point to the basal signal (at 0 s). $\boldsymbol{B}$, Fluorescence of Fluo-4 AM, indicative of calcium concentration, in cortical neurons is shown before AMPA treatment, after AMPA treatment (top panel), and after the combination of AMPA and NBQX treatment (bottom panel). C, GluR1 siRNA, Flag-Hes-1WT plasmid, Flag-Hes-15263A mutant plasmid, Hes-1 siRNA, or combined Hes-1 siRNA and GluR1 siRNA was cotransfected with pCMV-DsRED plasmid to cultured cortical neurons, and their effects on calcium fluorescence before and after AMPA treatment were shown. D, HA-JNK1 plasmid, sh-JNK1 (here we used sh-JNK1-2 from Fig. 5), or various combinations of the HA-JNK1 and Hes-1 plasmid, together with Hes-1 siRNA or GluR1 siRNA, was cotransfected with pCMV-DsRED plasmid to cultured cortical neurons, and their effects on calcium fluorescence before and after AMPA treatment are shown. Cell(s) indicated by arrow(s) and cells showed orange color in the merged images are cells successfully transfected with the desired plasmid and/or siRNA. The number of cells ranged from 25 to 45 for all experiments. E, Summary of effects of various plasmid and/or siRNA transfections on calcium fluorescence intensity. $\Delta F / F_{0}$ represents the ratio of fluorescence signal subtraction of basal signal over that of basal signal. It is then expressed as the ratio of fluorescence signal of transfected neurons over that of the neighboring nontransfected neurons. Experiments are in duplicate. The number of cells ranged from 15 to 20 for each group. Data are expressed as mean \pm SEM. ${ }^{*} p<0.05$, ${ }^{* *} p<0.01$, and ${ }^{\#} p<0.001$. 
Representative images for all the transfections are shown in Figure $12 C$, and the successfully transfected cell is indicated by an arrow. Results revealed that cells transfected with GluR1 siRNA showed reduced fluorescence intensity compared with that of the neighboring cells (Fig. 12C, top panel). The cell transfected with Hes-1WT plasmid also showed decreased fluorescence intensity (Fig. 12C, second panel). The fluorescence intensity for cells transfected with Hes-1S263A is higher than that of Hes-1WTtransfected cell (with the fluorescence intensity of the corresponding neighboring cells is similar) (Fig. 12C, middle panel). But for the cell transfected with Hes-1 siRNA, the fluorescence intensity was higher compared with that of the neighboring cells (Fig. 12C, fourth panel). Furthermore, the enhancing effect of Hes-1 siRNA on calcium fluorescence intensity was blocked when GluR1 siRNA was cotransfected (Fig. 12C, bottom panel). The quantification results are summarized in Figure $12 E(p<0.05, p<$ 0.01 , or $p<0.001$ for various comparisons).

In the second series of experiments, we examined the roles of JNK1 and Hes-1 involved in calcium influx. The result showed that, for the cell successfully transfected with the JNK1 plasmid, the fluorescence intensity was lower compared with that of the neighboring cells (Fig. 12D, top panel). But for the cell transfected with sh-JNK1, the fluorescence intensity was higher than that of the neighboring cells (Fig. 12D, second panel). However, this effect of sh-JNK1 was blocked when sh-JNK1 was cotransfected with the Hes-1WT plasmid (Fig. 12D, middle panel). In addition, the decreasing effect of JNK1 on calcium fluorescence intensity was blocked when Hes-1 siRNA was cotransfected (Fig. 12D, fourth panel). Conversely, the enhancing effect of sh-JNK1 on calcium fluorescence intensity was also blocked when GluR1 siRNA was transfected (Fig. 12D, bottom panel). The quantification results are summarized in Figure $12 E(p<0.05, p<0.01$, or $p<$ 0.001 for various comparisons).

\section{Discussion}

The GluR1 subunit of AMPA receptor is believed to play an important role in synaptic function (Mack et al., 2001; Schmitt et al., 2005). GluR1 expression is particularly important for the expression of LTP (Zamanillo et al., 1999). But most research studied the phosphorylation and trafficking of GluR1 involved in synaptic function; relatively less is known about the regulation mechanism for GluR1 expression. In this study, we have examined the signaling pathway and molecular mechanism that regulate GluR1 promoter activity, GluR1 expression, and GluR1-mediated calcium influx in cultured cortical neurons. Our results revealed that GluR1 is directly and negatively regulated by Hes-1. Hes-1 suppressed GluR1 promoter activity through direct binding to the N-box of GluR1 promoter, and each $\mathrm{N}$-box plays an equally important role in mediating the suppressing effect of Hes-1. In addition, Hes- 1 also AMPA in cortical neurons.

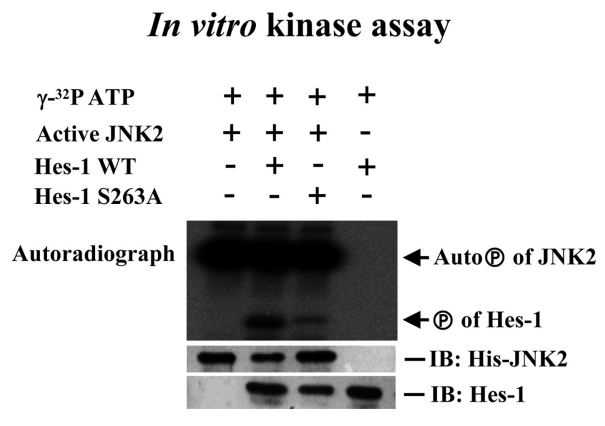

Figure 13. JNK2 also phosphorylates Hes-1 at Ser-263. In vitro kinase assay for JNK2 phosphorylation of Hes- 1 at Ser-263 was performed. Experiments are in duplicate.

\section{Stimulus (e.g. oxidative stress)}

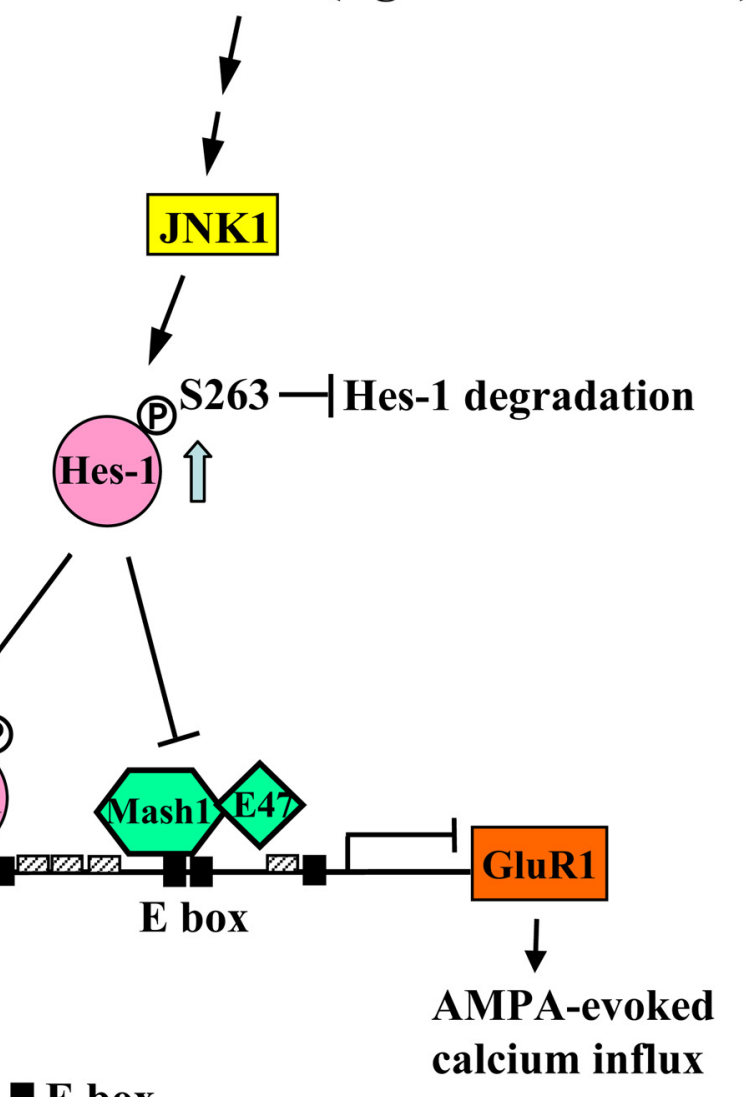

Figure 14. A schematic diagram showing the relationships among JNK activation, Hes-1 expression and phosphorylation, GluR1 expression, and AMPA-evoked calcium influx in cortical neurons. A stimulus, such as oxidative stress, would activate JNK1 signaling. JNK1 activation directly phosphorylates Hes-1 at Ser-263. Hes-1 phosphorylation at this residue results in stabilization of the Hes-1 protein. Upon direct binding to the N-boxes of the GluR1 promoter and through preventing Mash1/E47 complex from binding to the E-boxes of the GluR1 promoter, Hes-1 decreased GluR1 expression that leads to decreased calcium influx evoked by

suppressed GluR1 promoter activity through preventing Mash1/E47 complex from binding to the E-box of GluR1 promoter. In a previous study, Hes-1 was shown to suppress the promoter activity of the MAP2 gene also through direct binding to the N-box of the MAP2 promoter that is involved in melanoma progression, and this effect is Notch signaling dependent (Bhat et al., 2006). Because MAP2 is known to stabilize microtubules and is important for neurite outgrowth (Dehmelt et al., 2003), this report suggests that Hes-1 may be implicated in neuronal function. Here, we provide direct evidence that Hes- 1 suppressed 
GluR1 promoter activity and decreased GluR1 protein expression in cultured cortical neurons. Furthermore, Hes- 1 inhibition of GluR1 expression plays a role in suppressing calcium influx evoked by AMPA stimulation. This is the first report showing that Hes- 1 could regulate synaptic function in differentiated neurons, and this result is congruent with a previous report showing that Hes-5 impairs contextual fear memory in rats (Lee et al., 2007). More importantly, we have found that Hes- 1 inhibition of GluR1 expression is independent of Notch signaling. This latter result suggests that other signaling pathways mediate the effect of Hes-1 on GluR1 expression.

Hes- 1 was previously found to contain the protein kinase C consensus sites and phosphorylation of these sites inhibits the DNA binding activity of Hes-1 that is involved in nerve growth factormediated neurite outgrowth in PC12 cells (Ström et al., 1997). In another study, Hes-1 was found phosphorylated by calcium/ calmodulin-dependent kinase II $\delta$ (CaMKII $\delta$ ), and CaMKII $\delta$ phosphorylation of Hes- 1 alters the function of Hes- 1 from a repressor to an activator required for neuronal differentiation (Ju et al., 2004). Furthermore, Hes- 1 could be regulated by JNK signaling in human endothelial cells, and this is Notch signaling independent (Curry et al., 2006). But this study did not reveal the molecular mechanism underlying JNK1 regulation of Hes-1 expression. In a more recent study, JNK was found to phosphorylate the GluR2L and GluR4 subunits of AMPA receptor directly, and JNK phosphorylation of GluR2L regulates AMPA receptor trafficking following neuronal activation in a rapid and bidirectional manner (Thomas et al., 2008). But whether JNK also regulates GluR1 and the relationship among JNK, Hes-1, and GluR1 was not known. Our results provide the first evidence that JNK1 suppressed GluR1 promoter activity and GluR1 expression through the mediation of Hes- 1 in cultured cortical neurons. We also identified Hes-1 as a novel substrate of JNK1 and JNK1 phosphorylation of Hes- 1 stabilized the Hes- 1 protein. This result is consistent with other reports showing that JNK1 could also stabilize Sp1 and p53 as a consequence of JNK1 phosphorylation of these proteins (Fuchs et al., 1998; Chuang et al., 2008). These results also suggest that one common mechanism for JNK1 to stabilize a protein is mediated through JNK1 phosphorylation of this substrate protein. These results are also congruent with the report that JNK1 activity is present in the nucleus (Björkblom et al., 2005). But our results do not exclude the possibility that JNK1 may also upregulate and stabilize Hes-1 through other yet-unidentified mechanisms. Furthermore, Hes- 1 expression in cortical neurons and Hes- 1 suppression of GluR1 expression is independent of Notch signaling. This result is congruent with the report that JNK regulation of Hes-1 expression in endothelial cells is also independent of Notch (Curry et al., 2006). In addition, transforming growth factor- $\alpha$ was found to regulate Hes-1 expression through Ras/MAPK signaling in neuroblastoma cells that is also independent of Notch signaling (Stockhausen et al., 2005). Moreover, some inflammatory stimuli were found to increase Notch expression but decrease Hes-1 transcription in microglial cells (Grandbarbe et al., 2007). It is apparent that Hes-1 expression is regulated in both a Notch-dependent and Notch-independent manner. We have further found that, in addition to JNK1, JNK2 also directly phosphorylates Hes-1 at Ser-263 (Fig. 13). Thus, Hes-1 may be a common substrate of the JNK kinase family in neurons. This speculation is consistent with the report that bFGF2 enhances Hes-1 expression through direct binding of JNK-activating transcription factor 2 (ATF2) to the Hes-1 promoter (Sanalkumar et al., 2010), whereas ATF2 is a downstream target of JNK (Gupta et al., 1995). Moreover, Hes-1 suppression of GluR1 expression decreased calcium influx upon AMPA stimulation in cortical neurons. Hes-1 is well known to regulate neuronal differentiation and development.
Here, we provide the first evidence that Hes- 1 also regulates synaptic function in differentiated neurons. Whether Hes-1 also regulates other forms of neuronal plasticity awaits further investigation.

The regulation mechanism for GluR1 expression is barely known in the literature. In this study, we found that JNK1 negatively regulates GluR1 expression, and JNK1 inhibition of GluR1 expression decreased calcium influx evoked by AMPA stimulation in cortical neurons. This result suggests that JNK1 signaling decreases synaptic function, and this result is consistent with the finding that JNK mediates the impairing effect of amyloid- $\beta$ on LTP (Costello and Herron, 2004). It is also consistent with the report that JNK is involved in the inhibitory effect of IL- $1 \beta$ on LTP (Curran et al., 2003). Although the percentage of GluR1 receptor present at synapses is relatively low, we have demonstrated that both JNK1 and Hes-1 also suppressed GluR1 expression at synapses and synaptic membrane, and Hes- 1 also mediated the suppressing effect of JNK1 on GluR1, similar to their suppressing effect on GluR1 observed in the soma. These results are congruent with the report that GluR1 knock-out mice show impaired LTP (Mack et al., 2001) and indicate that synaptic GluR1 is functionally significant in addition to its regulation of AMPA-evoked calcium influx. In addition to suppression of GluR1 expression, JNK also regulates AMPA receptor trafficking. It is found that activation of Rap2, a small GTPase related to Rap1, inhibits AMPA receptor-mediated synaptic transmission for depotentiation through activation of JNK at hippocampal CA1 neurons (Zhu et al., 2005). Furthermore, JNK1 is also involved in AMPA receptor internalization in the nucleus accumbens after cocaine challenge in sensitized rats (Boudreau et al., 2007). Moreover, because JNK2/3 signaling is activated upon stress and neuropathological stimulation (Bogoyevitch and Kobe, 2006), and both JNK1 and JNK2 phosphorylate Hes-1 at Ser-263, the present result is also consistent with the finding that chronic mild stress decreases GluR1 expression in rat prefrontal cortex and hippocampus (Toth et al., 2008). Whether stress also upregulates Hes- 1 expression and decreases calcium influx requires further investigation.

In the present study, by using a very low dose of anisomycin (100 ng/ml) to activate JNK1, we found that it rapidly increased Hes-1 mRNA level within $1 \mathrm{~h}$, but anisomycin turns to decrease Hes-1 mRNA level at later time points (Fig. 7A). This is possibly due to negative autoregulation of Hes-1 expression because the Hes-1 promoter contains three N-boxes and Hes- 1 was found to bind its own promoter through these N-boxes to suppress its gene expression (Takebayashi et al., 1994).

In summary, in the present study, we have identified Hes- 1 as a novel substrate of JNK1. JNK1 phosphorylation of Hes-1 at Ser-263 stabilizes the Hes-1 protein. However, the regulation mechanism for GluR1 expression is rarely studied. We first demonstrated that Hes-1 could downregulate GluR1 expression through direct binding to the N-box and through preventing the Mash1/E47 complex from binding to the E-box of the GluR1 promoter, and this regulation is Notch independent. Moreover, we found that JNK1 decreased GluR1 expression through the mediation of Hes-1 and this signaling pathway inhibits calcium influx evoked by AMPA in cultured cortical neurons (Fig. 14). This is not only the first study revealing that JNK1 signaling could regulate GluR1 expression involved in synaptic function, this is also the first study showing a novel role of Hes-1 involved in synaptic function in differentiated neurons.

\section{References}

Bhat KM, Maddodi N, Shashikant C, Setaluri V (2006) Transcriptional regulation of human MAP2 gene in melanoma: role of neuronal bHLH factors and Notch1 signaling. Nucleic Acids Res 34:3819-3832. 
Björkblom B, Ostman N, Hongisto V, Komarovski V, Filén JJ, Nyman TA, Kallunki T, Courtney MJ, Coffey ET (2005) Constitutively active cytoplasmic c-Jun N-terminal kinase 1 is a dominant regulator of dendritic architecture: role of microtubule-associated protein 2 as an effector. J Neurosci 25:6350-6361.

Bogoyevitch MA, Kobe B (2006) Uses for JNK: the many and varied substrates of the c-Jun N-terminal kinases. Microbiol Mol Biol Rev 70:1061-1095.

Borges K, Dingledine R (2001) Functional organization of the GluR1 glutamate receptor promoter. J Biol Chem 276:25929-25938.

Boudreau AC, Reimers JM, Milovanovic M, Wolf ME (2007) Cell surface AMPA receptors in the rat nucleus accumbens increase during cocaine withdrawal but internalize after cocaine challenge in association with altered activation of mitogen-activated protein kinases. J Neurosci 27:10621-10635.

Chang L, Karin M (2001) Mammalian MAP kinase signalling cascades. Nature 410:37-40.

Chuang JY, Wang YT, Yeh SH, Liu YW, Chang WC, Hung JJ (2008) Phosphorylation by c-Jun $\mathrm{NH}_{2}$-terminal kinase 1 regulates the stability of transcription factor Sp1 during mitosis. Mol Biol Cell 19:1139-1151.

Costello DA, Herron CE (2004) The role of c-Jun N-terminal kinase in the $\mathrm{A} \beta$-mediated impairment of LTP and regulation of synaptic transmission in the hippocampus. Neuropharmacology 46:655-662.

Curran BP, Murray HJ, O'Connor JJ (2003) A role for c-Jun N-terminal kinase in the inhibition of long-term potentiation by interleukin-1beta and long-term depression in the rat dentate gyrus in vitro. Neuroscience 118:347-357.

Curry CL, Reed LL, Nickoloff BJ, Miele L, Foreman KE (2006) Notchindependent regulation of Hes- 1 expression by c-Jun $\mathrm{N}$-terminal kinase signaling in human endothelial cells. Lab Invest 86:842-852.

Davis RJ (2000) Signal transduction by the JNK group of MAP kinases. Cell 103:239-252.

Dehmelt L, Smart FM, Ozer RS, Halpain S (2003) The role of microtubuleassociated protein $2 \mathrm{c}$ in the reorganization of microtubules and lamellipodia during neurite initiation. J Neurosci 23:9479-9490.

Dingledine R, Borges K, Bowie D, Traynelis SF (1999) The glutamate receptor ion channels. Pharmacol Rev 51:7-61.

Fosbrink M, Aye-Han NN, Cheong R, Levchenko A, Zhang J (2010) Visualization of JNK activity dynamics with a genetically encoded fluorescent biosensor. Proc Natl Acad Sci U S A 107:5459-5464.

Frey U, Krug M, Reymann KG, Matthies H (1988) Anisomycin, an inhibitor of protein synthesis, blocks late phases of LTP phenomena in the hippocampal CA1 region in vitro. Brain Res 452:57-65.

Fuchs SY, Adler V, Pincus MR, Ronai Z (1998) MEKK1/JNK signaling stabilizes and activates p53. Proc Natl Acad Sci U S A 95:10541-10546.

Grandbarbe L, Michelucci A, Heurtaux T, Hemmer K, Morga E, Heuschling P (2007) Notch signaling modulates the activation of microglial cells. Glia 55:1519-1530.

Gupta S, Campbell D, Dérijard B, Davis RJ (1995) Transcription factor ATF2 regulation by the JNK signal transduction pathway. Science 267:389-393.

Gurd JW, Jones LR, Mahler HR, Moore WJ (1974) Isolation and partial characterization of rat brain synaptic plasma membranes. J Neurochem 22:281-290.

Hollmann M, Heinemann S (1994) Cloned glutamate receptors. Annu Rev Neurosci 17:31-108.

Humeau Y, Reisel D, Johnson AW, Borchardt T, Jensen V, Gebhardt C, Bosch V, Gass P, Bannerman DM, Good MA, Hvalby Ø, Sprengel R, Lüthi A (2007) A pathway-specific function for different AMPA receptor subunits in amygdala long-term potentiation and fear conditioning. J Neurosci 27:10947-10956.

Iordanov MS, Pribnow D, Magun JL, Dinh TH, Pearson JA, Chen SL, Magun BE (1997) Ribotoxic stress response: activation of the stress-activated protein kinase JNK1 by inhibitors of the peptidyl transferase reaction and by sequence-specific RNA damage to the $\alpha$-sarcin/ricin loop in the $28 \mathrm{~S}$ rRNA. Mol Cell Biol 17:3373-3381.

Ishibashi M, Moriyoshi K, Sasai Y, Shiota K, Nakanishi S, Kageyama R (1994) Persistent expression of helix-loop-helix factor HES-1 prevents mammalian neural differentiation in the central nervous system. EMBO J 13:1799-1805.

Ishibashi M, Ang SL, Shiota K, Nakanishi S, Kageyama R, Guillemot F (1995) Targeted disruption of mammalian hairy and Enhancer of split homolog-1 (HES-1) leads to up-regulation of neural helix-loop-helix factors, premature neurogenesis, and severe neural tube defects. Genes Dev 9:3136-3148.
Ju BG, Solum D, Song EJ, Lee KJ, Rose DW, Glass CK, Rosenfeld MG (2004) Activating the PARP-1 sensor component of the groucho/TLE1 corepressor complex mediates a CaMKinase IIdelta-dependent neurogenic gene activation pathway. Cell 119:815-829.

Kageyama R, Ohtsuka T (1999) The Notch-Hes pathway in mammalian neural development. Cell Res 9:179-188.

Kageyama R, Ohtsuka T, Hatakeyama J, Ohsawa R (2005) Roles of bHLH genes in neural stem cell differentiation. Exp Cell Res 306:343-348.

Kageyama R, Ohtsuka T, Kobayashi T (2008) Roles of Hes genes in neural development. Dev Growth Differ 50 [Suppl 1]:S97-S103.

Lee CT, Ma YL, Lee EH (2007) Serum- and glucocorticoid-inducible kinasel enhances contextual fear memory formation through downregulation of the expression of Hes5. J Neurochem 100:1531-1542.

Mack V, Burnashev N, Kaiser KM, Rozov A, Jensen V, Hvalby O, Seeburg PH, Sakmann B, Sprengel R (2001) Conditional restoration of hippocampal synaptic potentiation in Glur-A-deficient mice. Science 292:2501-2504.

Makino H, Malinow R (2009) AMPA receptor incorporation into synapses during LTP: the role of lateral movement and exocytosis. Neuron 64:381-390.

Malinow R, Malenka RC (2002) AMPA receptor trafficking and synaptic plasticity. Annu Rev Neurosci 25:103-126.

Pickering C, Gustafsson L, Cebere A, Nylander I, Liljequist S (2006) Repeated maternal separation of male Wistar rats alters glutamate receptor expression in the hippocampus but not the prefrontal cortex. Brain Res 1099:101-108.

Ross SE, Greenberg ME, Stiles CD (2003) Basic helix-loop-helix factors in cortical development. Neuron 39:13-25.

Sanalkumar R, Indulekha CL, Divya TS, Divya MS, Anto RJ, Vinod B, Vidyanand S, Jagatha B, Venugopal S, James J (2010) ATF2 maintains a subset of neural progenitors through CBF1/Notch independent Hes-1 expression and synergistically activates the expression of Hes-1 in Notchdependent neural progenitors. J Neurochem 113:807-818.

Sasai Y, Kageyama R, Tagawa Y, Shigemoto R, Nakanishi S (1992) Two mammalian helix-loop-helix factors structurally related to Drosophila hairy and Enhancer of split. Genes Dev 6:2620-2634.

Schmitt WB, Sprengel R, Mack V, Draft RW, Seeburg PH, Deacon RM, Rawlins JN, Bannerman DM (2005) Restoration of spatial working memory by genetic rescue of GluR-A-deficient mice. Nat Neurosci 8:270-272.

Shi S, Hayashi Y, Esteban JA, Malinow R (2001) Subunit-specific rules governing AMPA receptor trafficking to synapses in hippocampal pyramidal neurons. Cell 105:331-343.

Stockhausen MT, Sjölund J, Axelson H (2005) Regulation of the Notch target gene Hes-1 by TGFalpha-induced Ras/MAPK signaling in human neuroblastoma cells. Exp Cell Res 310:218-228.

Ström A, Castella P, Rockwood J, Wagner J, Caudy M (1997) Mediation of NGF signaling by post-translational inhibition of HES-1, a basic helix-loop-helix repressor of neuronal differentiation. Genes Dev 11:3168-3181.

Tai DJ, Hsu WL, Liu YC, Ma YL, Lee EH (2011) Novel role and mechanism of protein inhibitor of activated STAT1 in spatial learning. EMBO J 30:205-220

Takebayashi K, Sasai Y, Sakai Y, Watanabe T, Nakanishi S, Kageyama R (1994) Structure, chromosomal locus, and promoter analysis of the gene encoding the mouse helix-loop-helix factor HES-1. Negative autoregulation through the multiple N box elements. J Biol Chem 269:5150-5156.

Thomas GM, Lin DT, Nuriya M, Huganir RL (2008) Rapid and bidirectional regulation of AMPA receptor phosphorylation and trafficking by JNK. EMBO J 27:361-372.

Toth E, Gersner R, Wilf-Yarkoni A, Raizel H, Dar DE, Richter-Levin G, Levit O, Zangen A (2008) Age-dependent effects of chronic stress on brain plasticity and depressive behavior. J Neurochem 107:522-532.

Yang YC, Lin CH, Lee EH (2006) Serum- and glucocorticoid-inducible kinase 1 (SGK1) increases neurite formation through microtubule depolymerization by SGK1 and by SGK1 phosphorylation of tau. Mol Cell Biol 26:8357-8370.

Zamanillo D, Sprengel R, Hvalby O, Jensen V, Burnashev N, Rozov A, Kaiser KM, Köster HJ, Borchardt T, Worley P, Lübke J, Frotscher M, Kelly PH, Sommer B, Andersen P, Seeburg PH, Sakmann B (1999) Importance of AMPA receptors for hippocampal synaptic plasticity but not for spatial learning. Science 284:1805-1811.

Zhu Y, Pak D, Qin Y, McCormack SG, Kim MJ, Baumgart JP, Velamoor V, Auberson YP, Osten P, van Aelst L, Sheng M, Zhu JJ (2005) Rap2-JNK removes synaptic AMPA receptors during depotentiation. Neuron 46: 905-916. 